\title{
Optimal control for fermentative production of fructo-oligosaccharides in fed-batch bioreactor ${ }^{\text {is }}$
}

\author{
J. Schorsch ${ }^{\mathrm{a}, *}$, C.C. Castro ${ }^{\mathrm{b}}$, L.D. Couto ${ }^{\mathrm{a}}$, C. Nobre ${ }^{\mathrm{c}}$, M. Kinnaert ${ }^{\mathrm{a}}$ \\ a Université libre de Bruxelles (ULB), SAAS Department CP 165/55, B-1050 Bruxelles, Belgium \\ ${ }^{\mathrm{b}}$ University of Mons, Applied Chemistry and Biochemistry Department, Biosciences Institute, 56, Rue de l'Epargne, 7000 Mons, Belgium \\ ' Centre of Biological Engineering, University of Minho, Campus de Gualtar, 4710-057 Braga, Portugal
}

\section{A R T I C L E I N F O}

\section{Article history:}

Received 30 June 2017

Received in revised form 28 February 2019

Accepted 12 March 2019

\section{Keywords:}

Optimal control

Maximum principle of Pontryagin

Fructo-oligosaccharides

Fed-batch bioreactor

\begin{abstract}
A B S T R A C T
The production of high-content fructo-oligosaccharides by fermentation in a fed-batch bioreactor is considered. Fed-batch scenario allows to reduce inhibition mechanisms caused by the presence of byproducts, such as glucose, while maximizing biomass production. The determination of the feed flow rate profile that maximizes the concentration of fructo-oligosaccharides is performed by resorting to the maximum principle of Pontryagin. The optimization procedure takes into account inequality constraints on the state and control variables and allows the determination of the unspecified final fermentation time corresponding to maximal fructo-oligosaccharides content. The performance of the approach is demonstrated by simulations in the case of fructo-oligosaccharide production by Aspergillus sp.
\end{abstract}

(c) 2019 Elsevier Ltd. All rights reserved.

\section{Introduction}

Fructo-oligosaccharides (FOS), such as 1-kestose $\left(\mathrm{GF}_{2}\right)$, nystose $\left(\mathrm{GF}_{3}\right)$ and 1-fructofuranosyl nystose $\left(\mathrm{GF}_{4}\right)$, are used as food ingredients due to their prebiotic properties that stimulate human health. They selectively increase the probiotic bacteria development resulting in the prevention of many gastrointestinal diseases, colorectal cancer, diabetes, osteoporosis, etc [1,2]. Moreover, FOS taste profile and rheology are similar to sucrose, with a $30 \%$ relative sweetness [3] which allows its use as a low-caloric replacement of sugar in dietetic and diabetic food. FOS are found naturally in many different sources, including fruits, vegetables and honey, although they are season-limited and usually present in low amounts. Industrially, FOS are produced by transfructosylation of sucrose (GF) (a disaccharide composed by the monosaccharides glucose $(G)$ and fructose $(F))$ through microbial enzymes, namely fructosyltransferase (FTase) (EC 2.4.1.9) and $\beta$-fructofuranosidase (FFase) (EC 3.2.1.26), naturally present in many microorganisms. Distinct enzymatic activities of these specific enzymes can be obtained from different microorganisms, including Aureobasidium pullulans [4] or Aspergillus sp. [5].

\footnotetext{
is Julien Schorsch and Michel Kinnaert thank the F.R.S.-FNRS, the Belgium National Fund for Scientific Research (PDR: T.0196.13) for its financial support.

* Corresponding author.

E-mail addresses: julien.schorsch@gmail.com (J. Schorsch), clarissenobre@deb.uminho.pt (C. Nobre), michel.kinnaert@ulb.ac.be (M. Kinnaert).
}

FOS production through fermentation using microorganisms whole cells, which in turn produce enzymes with transfructosylating activity, significantly depends on the bioreactor operational conditions and concentrations of the generated by-products $[4,6,7]$. Temperature, $\mathrm{pH}$, agitation speed and oxygen flow rate are some of the bioreactor operational variables that can affect the chemical reactions during the enzymatic activities. Adequate values should then be determined to optimize the reaction conditions. Batch experiments have been carried out to produce FOS and have shown enhanced results using a pH 5-6 and a temperature range of $30-45^{\circ} \mathrm{C}$. Concerning the agitation speed and the oxygen flow rate in the case of FOS production by extracted and purified enzymes, FOS concentration is improved for a speed of $550 \mathrm{rpm}$ and an oxygen flow rate of $0.7 \mathrm{Lmin}^{-1}$ [7]. In the case of FOS produced by whole cells a lower agitation of $200 \mathrm{rpm}$ is required.

Moreover, FOS production dynamics by microbial cells can be responsive to different factors, including cells growth, metabolic changes caused by a depletion or augmentation of substrates, as well as changes in the overall metabolism, such as changes in uptake rates, depending on substrate concentration [8]. Since sucrose is the FOS-producing substrate, its initial concentration is treated as a user-parameter and thus easy to set. Therefore, the focus is mostly placed on the glucose, which appears in the fermentation mixture as the result of the $\beta$-fructofuranosidase activity. A high level of glucose can inhibit the fructosyl-transferring reaction [6,9] and so, affect FOS synthesis. Glucose control can improve FOS yields and purity, while increasing the prebiotic activity of the final mixture. In addition, it can improve the further separation and 


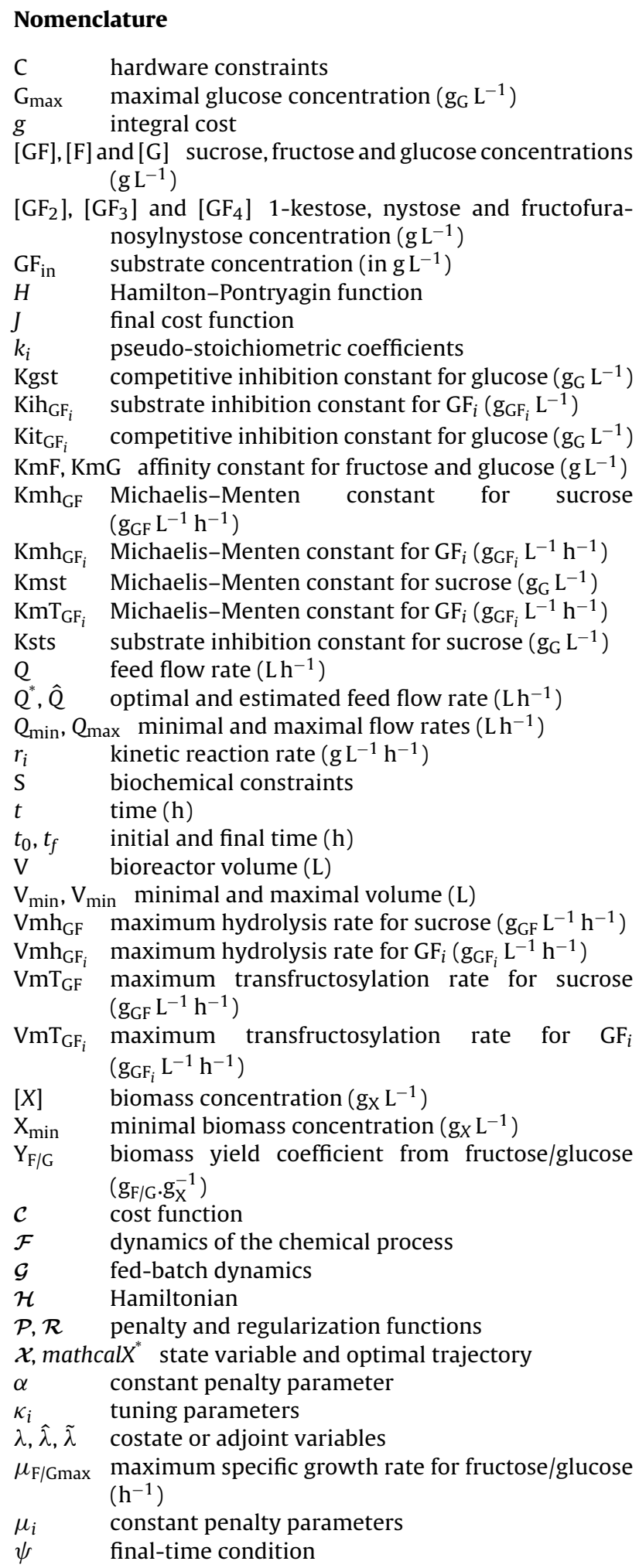

purification of FOS, industrially performed by simulated-movingbed chromatography [10].

Different strategies have been applied for the reduction of the glucose in the fermentation mixture [11]. These include ultra and nano filtration [12], activated charcoal systems [13], ion-exchange chromatography [14] or microbial treatment [10]. Herein a fedbatch fermentation procedure is considered in which a controlled substrate feeding profile can provide the regulation of the glucose concentration in the mixture. Monitoring feeding of the substrate allows to overcome the substrate inhibitory issues (mainly related to the osmotic stress), and it can also restrict the cells growth rate and so, avoid the limitations in oxygen and heat transfer. Under these circumstances, the FOS concentration and productivity can be maximized, while the accumulation of by-products can be prevented.

Regarding the bioreactor fermentation control, two distinct control design methods can be generally applied [15]: (i) the first uses a control scheme, i.e., a profile of the substrate feeding rate, that does not resort to a mathematical model; the initial trajectories obtained in the batch case determine the fed-batch or semi-batch operation. However, this method is not able to take in consideration the mechanisms that can interfere with the enzymatic activity, such as the presence of some by-products in the reaction mixture during FOS production; (ii) the second is based on a mathematical model representing the cell growth and the biochemical reactions responsible for FOS synthesis and hydrolysis. In this context, a fermentative model is selected based on the internal state of the growing cells or on an unstructured model such as the Monod law. The hydrolysis reactions, as well as the transfructosylation reactions are simulated by the Michaelis-Menten law. From these two control strategies, only the one that does not resort to a mathematical model (i) has been used for FOS production. This paper focuses on the second approach (ii).

Concerning the FOS production process, few papers considering the mathematical modeling have been published [5,16-18]. In the present paper, the mathematical model studied in $[5,17]$ is exploited to determine an optimal profile of the substrate feed rate in fed-batch bioreactor process.

Determining an optimal substrate feed rate for fed-batch bioreactor, according to a mathematical model, is one of the main challenges in chemical engineering science (see e.g. $[15,19,20])$. The feed rate concentration profile of the fed-batch cultivation implies the adjustment of different issues, including: feeding starting time, substrate concentration, feeding-rate and time to stop feeding. As it will be shown in the problem statement, the maximization of FOS production turns out to be a singular optimal control problem which requires specific approaches to avoid numerical ill-conditioning.

For solving such a problem, two types of approaches can be distinguished: indirect or variational approaches and direct transcription approaches [21]. The former ones exploit the first order necessary optimality conditions resulting from Pontryagin's maximum principle to deduce a two point boundary value problem (BVP) which can be solved by resorting to different techniques. Here a control vector iteration approach is used. It solves repeatedly the state and adjoint equations associated to the optimal control problem until convergence is reached. State and input inequality constraints are handled by penalty functions [22]. The direct transcription approaches consist in performing full discretization of the control and state trajectories, as well as the state equations. The optimization problem then takes the form of a large nonlinear program which can be solved by resorting to large scale nonlinear programming solvers. However, as the considered optimal control problem is singular, care must be taken to choose of the control profile in order to avoid ill-conditioned behaviors [21]. A very small time step should be applied to obtain an accurate control profile, which in the same time may involve ill-conditionning of the Hessian matrix. The use of a regularization term in the cost function is a way to avoid this ill-conditioning but requires proper tuning of the weight of the regularization term. The approaches relying on genetic algorithms proposed in $[23,24]$ can be seen as direct transcription approaches and are based on probabilistic search procedure. Such algorithms suffer from requiring a good knowledge 
of the model for a proper initialization while not providing any guarantee that the optimal solution is reached.

In this paper we have chosen to apply the first class of method since we believe they provide more insight into the solution of the optimal control problem.

The paper is organized as follows. In Section 2, the bioreactor model and the associated mathematical equations are described, followed by the presentation of the constraints and the FOS optimization problem. Section 3 deals with penalty functions to ensure the convexity of the problem and with Pontryagin's maximum principle. Section 4 shows the performance of the method through several simulations and provides an analysis of the solution.

\section{Modeling and problem statement}

The main biochemical reactions involved in the fermentative production of FOS are first presented below. Next, a mathematical model describing this process in a fed-batch scenario is determined. Inequality constraints to reduce the inhibition of FOS production due to a high glucose concentration and a low enzyme activity are then introduced before stating the optimal control problem.

\subsection{Biochemical reactions}

The chemical processes representing the fermentative production of FOS in the bioreactor are described by hydrolysis reactions of sucrose and FOS by FFase given by

$G F \stackrel{r_{1}}{\rightarrow} k_{1} G+k_{2} F$

and

$\left\{\begin{array}{l}G F_{2} \stackrel{r_{2}}{\rightarrow} k_{3} G F+k_{4} F \\ G F_{3} \stackrel{r_{3}}{\rightarrow} k_{5} G F_{2}+k_{6} F \\ G F_{4} \stackrel{r_{4}}{\rightarrow} k_{7} G F_{3}+k_{8} F\end{array}\right.$

and by the synthesis reactions of FOS by FTase, given by

$\left\{\begin{array}{l}2 G F \stackrel{r_{5}}{\rightarrow} k_{9} G F_{2}+k_{10} G \\ 2 G F_{2} \stackrel{r_{6}}{\rightarrow} k_{11} G F_{3}+k_{12} G F \\ 2 G F_{3} \stackrel{r_{7}}{\rightarrow} k_{13} G F_{4}+k_{14} G F_{2}\end{array}\right.$

where $k_{i}$ for $i=1, \ldots, 14$ are the pseudo-stoichiometric coefficients (see Table 1 ).
The kinetic reaction rates $r_{i}\left(\right.$ in $\mathrm{L}^{-1} \mathrm{~h}^{-1}$ ) for $i=1, \ldots, 7$, are given by the Michaelis-Menten law augmented with or without substrate inhibition and/or a competitive glucose inhibition

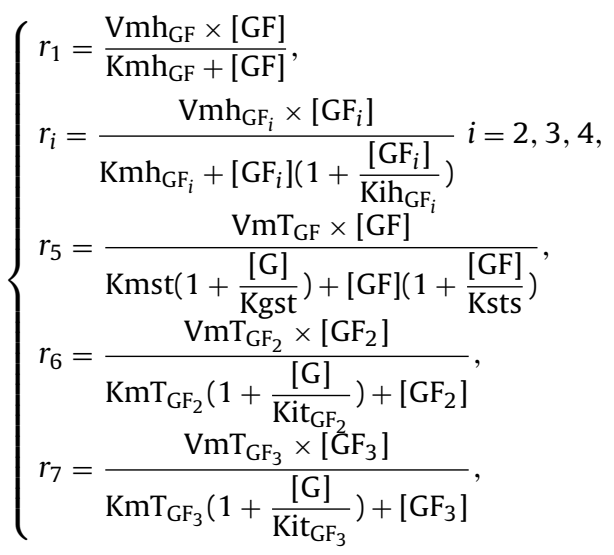

where $[\mathrm{GF}],[\mathrm{G}],[\mathrm{F}],\left[\mathrm{GF}_{2}\right],\left[\mathrm{GF}_{3}\right]$ and $\left[\mathrm{GF}_{4}\right]$ respectively represent the concentrations of sucrose, glucose, fructose, 1-kestose, nystose and 1-fructofuranosyl nystose (in $\mathrm{g} \mathrm{L}^{-1}$ ). Notice that the inhibition of 1-kestose and nystose by glucose is characterized in $r_{5}, r_{6}$ and $r_{7}$ by the terms Kgst, $\mathrm{Kit}_{\mathrm{GF}_{2}}$ and $\mathrm{Kit}_{\mathrm{GF}_{3}}$ which are the inhibitor dissociation constants. The constant model parameters are provided in Table 1.

The biochemical reactions associated to the biomass production are

$\left\{\begin{array}{l}Y_{G} G \stackrel{r_{8}}{\rightarrow} X \\ Y_{F} F \stackrel{r_{9}}{\rightarrow} X\end{array}\right.$

where $X$ represents the biomass and $Y_{G}$ and $Y_{F}$ (in $g_{G / F} \cdot g_{X}^{-1}$ ) correspond to the biomass yields when the glucose or fructose, respectively, are used for biomass growth. They are given by

$\mathrm{Y}_{\mathrm{G} / \mathrm{F}}=\frac{\text { MolecularweightofG } / \mathrm{F}}{\text { MolecularweightofX(unknown })}$.

The kinetic equations for $r_{8}$ and $r_{9}$ (in $\mathrm{g} \mathrm{L}^{-1} \mathrm{~h}^{-1}$ ) are given by the Monod law and represent the growth rate of the microorganism

$r_{8}=\frac{\mu_{\mathrm{Gmax}}[\mathrm{G}][\mathrm{X}]}{[\mathrm{G}]+\mathrm{KmG}}$ and $r_{9}=\frac{\mu_{\mathrm{Fmax}}[\mathrm{F}][\mathrm{X}]}{[\mathrm{F}]+\mathrm{KmF}}$,

where $[\mathrm{X}]$ is the biomass concentrations (in $\mathrm{g} \mathrm{L}^{-1}$ ).

\subsection{Mathematical model}

From the biochemical reactions stated in Eq. (1), a dynamic model describing the evolution of the component concentrations

Table 1

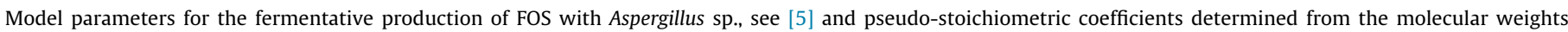
(operating conditions: temperature $30^{\circ} \mathrm{C}, \mathrm{pH} 5$ ).

\begin{tabular}{|c|c|c|c|c|c|c|c|}
\hline Parameter & Value & Units & Parameter & Value & Units & Parameter & Value \\
\hline $\mathrm{Vmh}_{\mathrm{GF}}$ & 1.43 & $\mathrm{~g}_{\mathrm{GF}} \mathrm{L}^{-1} \mathrm{~h}^{-1}$ & $\mathrm{KmG}$ & 397.98 & $\mathrm{~g}_{\mathrm{G}} \mathrm{L}^{-1}$ & $k_{1}$ & $180 / 342$ \\
\hline $\mathrm{Kmh}_{\mathrm{GF}}$ & 111.57 & $\mathrm{~g}_{\mathrm{GF}} \mathrm{L}^{-1}$ & $\mu_{\mathrm{Gmax}}$ & $2.89811 \mathrm{e}-5$ & $\mathrm{~h}^{-1}$ & $k_{2}$ & $180 / 342$ \\
\hline $\mathrm{VmT}_{\mathrm{GF}}$ & 49.99 & $\mathrm{~g}_{\mathrm{GF}} \mathrm{L}^{-1} \mathrm{~h}^{-1}$ & $\mathrm{KmF}$ & 11.45 & $\mathrm{~g}_{\mathrm{F}} \mathrm{L}^{-1}$ & $k_{3}$ & $342 / 504$ \\
\hline Ksts & 911.16 & $\mathrm{~g}_{\mathrm{GF}} \mathrm{L}^{-1}$ & $\mu_{\mathrm{Fmax}}$ & 0.0097 & $\mathrm{~h}^{-1}$ & $k_{4}$ & $180 / 504$ \\
\hline Kmst & 70.22 & $\mathrm{~g}_{\mathrm{GF}} \mathrm{L}^{-1}$ & $\mathrm{Y}_{\mathrm{G} / \mathrm{X}}$ & 29.23 & $g_{G} \cdot g_{X}^{-1}$ & $k_{5}$ & $504 / 666$ \\
\hline Kgst & 24.57 & $\mathrm{~g}_{\mathrm{G}} \mathrm{L}^{-1}$ & $\mathrm{Y}_{\mathrm{F} / \mathrm{X}}$ & 79.34 & $\mathrm{~g}_{\mathrm{F}} \cdot \mathrm{g}_{\mathrm{X}}^{-1}$ & $k_{6}$ & $180 / 666$ \\
\hline $\mathrm{Vmh}_{\mathrm{GF}_{2}}$ & 7.58 & $\mathrm{~g}_{\mathrm{GF}_{2}} \mathrm{~L}^{-1} \mathrm{~h}^{-1}$ & $\mathrm{VmT}_{\mathrm{GF}_{2}}$ & 41.63 & $\mathrm{~g}_{\mathrm{GF}_{2}} \mathrm{~L}^{-1} \mathrm{~h}^{-1}$ & $k_{7}$ & $666 / 828$ \\
\hline $\mathrm{Kih}_{\mathrm{GF}_{2}}$ & 2.72 & $\mathrm{~g}_{\mathrm{GF}_{2}} \mathrm{~L}^{-1}$ & $\mathrm{KmT}_{\mathrm{GF}_{2}}$ & 239.88 & $\mathrm{~g}_{\mathrm{GF}_{2}} \mathrm{~L}^{-1}$ & $k_{8}$ & $180 / 828$ \\
\hline $\mathrm{Kmh}_{\mathrm{GF}_{2}}$ & 0.61 & $\mathrm{~g}_{\mathrm{GF}_{2}} \mathrm{~L}^{-1}$ & $\mathrm{Kit}_{\mathrm{GF}_{2}}$ & 49.96 & $\mathrm{~g}_{\mathrm{G}} \mathrm{L}^{-1}$ & $k_{9}$ & $504 / 342$ \\
\hline $\mathrm{Vmh}_{\mathrm{GF}_{3}}$ & 7.97 & $\mathrm{~g}_{\mathrm{GF}_{3}} \mathrm{~L}^{-1} \mathrm{~h}^{-1}$ & $\mathrm{VmT}_{\mathrm{GF}_{3}}$ & 11.53 & $\mathrm{~g}_{\mathrm{GF}_{3}} \mathrm{~L}^{-1} \mathrm{~h}^{-1}$ & $k_{10}$ & $342 / 180$ \\
\hline $\mathrm{Kih}_{\mathrm{GF}_{3}}$ & 10.52 & $\mathrm{~g}_{\mathrm{GF}_{3}} \mathrm{~L}^{-1}$ & $\mathrm{KmT}_{\mathrm{GF}_{3}}$ & 333.07 & $\mathrm{~g}_{\mathrm{GF}_{3}} \mathrm{~L}^{-1}$ & $k_{11}$ & $666 / 504$ \\
\hline $\mathrm{Kmh}_{\mathrm{GF}_{3}}$ & 177.41 & $\mathrm{~g}_{\mathrm{GF}_{3}} \mathrm{~L}^{-1}$ & $\mathrm{Kit}_{\mathrm{GF}_{3}}$ & 49.95 & $\mathrm{~g}_{\mathrm{G}} \mathrm{L}^{-1}$ & $k_{12}$ & $342 / 504$ \\
\hline $\mathrm{Vmh}_{\mathrm{GF}_{4}}$ & 7.35 & $\mathrm{~g}_{\mathrm{GF}_{4}} \mathrm{~L}^{-1} \mathrm{~h}^{-1}$ & $\mathrm{Kmh}_{\mathrm{GF}_{4}}$ & 724.07 & $\mathrm{~g}_{\mathrm{GF}_{4}} \mathrm{~L}^{-1}$ & $k_{13}$ & $828 / 666$ \\
\hline $\mathrm{Kih}_{\mathrm{GF}_{4}}$ & 6.21 & $\mathrm{~g}_{\mathrm{GF}_{4}} \mathrm{~L}^{-1}$ & & & & $k_{14}$ & $504 / 666$ \\
\hline
\end{tabular}


has first been proposed in [18], and next in [16] where the nystose hydrolysis was added. The model has then been improved in [5] by including the 1-kestose and the 1-fructosylfuranosyl nystose hydrolysis as well as the biomass kinetic process represented in Eq. (3). The latter, a more complete model, in a fed-batch scenario, is herein presented. The evolution of the component concentrations is described by

$$
\left\{\begin{array}{l}
\frac{d \mathcal{X}(t)}{d t}=f(Q, \mathcal{X})=\mathcal{F}(\mathcal{X})+\mathcal{G}(\mathcal{X}) Q(t) \\
\mathcal{X}\left(t_{0}\right)=\mathcal{X}_{0} \\
\text { overthetimeinterval } t_{0} \leq t \leq t_{f} \text { and } Q(t) \in \mathbb{R}
\end{array}\right.
$$

$t_{0}$ and $t_{f}$ are respectively the known initial and the undetermined final time (in h). $f: \mathbb{R} \times \mathbb{R}_{+}^{8} \rightarrow \mathbb{R}_{+}^{8}$ is function of $Q$ and $\mathcal{X}$ involving the reaction rates, the pseudo-stochiometric coefficients and the concentrations defined below. The state variable vector $\mathcal{X}(t) \in \mathbb{R}_{+}^{8}$ is defined as

$\mathcal{X}^{\mathrm{T}}=\left([\mathrm{GF}][\mathrm{G}][\mathrm{F}]\left[\mathrm{GF}_{2}\right]\left[\mathrm{GF}_{3}\right]\left[\mathrm{GF}_{4}\right][\mathrm{X}] \mathrm{V}\right)$,

where $\mathrm{V}$ is the volume (in $\mathrm{L}$ ) of the solution in the bioreactor vessel. $\mathcal{X}_{0} \in \mathbb{R}_{+}^{8}$ is the initial state at $t_{0} \cdot Q(t) \in \mathbb{R}$ is the control representing the feed flow rate (in $\mathrm{Lh}^{-1}$ ) at time $t \in \mathbb{R}^{+}=[0,+\infty[$. Since the fedbatch case is considered in this paper, the model is subjected to the following final-time condition

$\psi\left(\mathcal{X}\left(t_{f}\right)\right)=\mathrm{V}\left(t_{f}\right)-\mathrm{V}_{\max } \equiv 0$

which determines the vessel volume at the final time $t_{f}, V_{\max }$ representing the effective maximal volume in $\mathrm{L}$. The function $\mathcal{F}$ characterizes the main dynamics of the chemical process

$$
\mathcal{F}(\mathcal{X})=\left(\begin{array}{c}
-r_{1}+k_{3} r_{2}-r_{5}+\frac{k_{12} r_{6}}{2} \\
k_{1} r_{1}+\frac{k_{10} r_{5}}{2}-\mathrm{Y}_{\mathrm{G}} r_{8} \\
k_{2} r_{1}+k_{4} r_{2}+k_{6} r_{3}+k_{8} r_{4}-\mathrm{Y}_{\mathrm{F}} r_{9} \\
-r_{2}+k_{5} r_{3}+\frac{k_{9} r_{5}}{2}-r_{6}+\frac{k_{14} r_{7}}{2} \\
-r_{3}+k_{7} r_{4}+\frac{k_{11} r_{6}}{2}-r_{7} \\
-r_{4}+\frac{k_{13} r_{7}}{2} \\
r_{8}+r_{9} \\
0
\end{array}\right),
$$

where each kinetic reaction rate is a function of $\mathcal{X}$ specified in Eqs. (2) and (4). $\mathcal{G}$ describes the fed-batch dynamics

$$
\mathcal{G}(\mathcal{X})=\frac{-1}{\mathrm{~V}}\left[\left([\mathrm{GF}]-\left[\mathrm{GF}_{\mathrm{in}}\right]\right) \quad[\mathrm{G}] \quad[\mathrm{F}] \quad\left[\mathrm{GF}_{2}\right] \quad\left[\mathrm{GF}_{3}\right] \quad\left[\mathrm{GF}_{4}\right][\mathrm{X}]-\mathrm{V}\right]^{\mathrm{T}}
$$

$\left[\mathrm{GF}_{\text {in }}\right]$ is the substrate concentration (in $\mathrm{g} \mathrm{L}^{-1}$ ), namely the sucrose concentration, in the feed.

\subsection{Inequality constraints}

The production of FOS is sensitive to experimental conditions such as $\mathrm{pH}$, agitation speed and temperature [6,25]. These conditions do not appear directly in the considered system (5) but are directly monitored and controlled by the bioreactor control unit. Here, we assume that adequate values have been chosen for these experimental conditions on the basis of [5], and we account for constraints on the biomass and the glucose concentrations as well as constraints on the substrate feed rate.

\subsubsection{Biochemical constraints}

As previously mentioned, FOS production through microbial transformation can be hampered by the presence of high concentrations of glucose in the medium, which can reduce the transfructosylating activity [7,26,27]. In addition, high cellular densities able to produce transfructosylating enzymes during a short cultivation time can improve FOS production. The amount of glucose released during the fermentation cannot be directly adjusted from the experimental initial conditions in batch mode. In contrast, the fed-batch scenario provides the means to control the amount of glucose, and therefore the FOS production. Thanks to the reaction rates $r_{5}, r_{6}$ and $r_{7}$, the developed model (5) takes into consideration glucose competitive inhibition. High glucose concentration implies large inhibition, which would result in less FOS production. To reduce the influence of the glucose inhibition in the model, a mathematical strategy is to add an inequality constraint limiting the glucose concentration evolution.

While substrate feed is pumped to the bioreactor, dilution effects occur affecting by-products and biomass. These effects allow the control of inhibition mechanisms caused by the by-products and the regulation of the biomass growth rate inside the bioreactor. Biomass regulation is necessary to ensure the required hydrolysis reactions and should be initially incorporated in the differential equations. However, the considered model determined in $[5,17]$ does not take this issue into account. Therefore the growth rate of the microorganism represented by the Monod equations $r_{8}$ and $r_{9}$ should be controlled to improve transfructosylating activity to produce FOS. This can be done by adding a constraint avoiding the biomass concentration to decrease below a certain level. Such constraint is thus added to keep a sufficient biomass level.

Let $\mathrm{X}_{\min }$ and $\mathrm{G}_{\max }$ (in $\mathrm{g} \mathrm{L}^{-1}$ ) denote the lowest required concentration of biomass and the maximal quantity of glucose, respectively, to avoid a strong enzyme inhibition during the chemical reactions; biochemical (or state) inequality constraints are summarized in $\mathrm{S}(\mathcal{X})$ by

$\mathrm{S}(\mathcal{X})=\left(\begin{array}{l}\mathrm{X}_{\mathrm{min}}-[\mathrm{X}](t) \\ {[\mathrm{G}](t)-\mathrm{G}_{\max }}\end{array}\right) \leq 0$ forall $t_{0} \leq t \leq t_{f}$.

\subsubsection{Hardware constraints}

The input flow rate $Q(t)$, namely control variable, containing nutrients at a concentration $\mathrm{GF}_{\text {in }}$, is limited by the feed pump capacity. Let $Q_{\max }$ and $Q_{\min }$ be the maximal and minimal flow rates (in $\mathrm{Lh}^{-1}$ ) respectively. The constraints on the control variable are therefore given by the vector of inequality constraints $C(Q)$

$C(Q)=\left(\begin{array}{l}Q_{\min }-Q(t) \\ Q(t)-Q_{\max }\end{array}\right) \leq 0$ forall $t_{0} \leq t \leq t_{f}$.

A piecewise continuous function $Q:\left[t_{0}, t_{f}\right] \rightarrow \mathbb{R}$ such that $Q(t)$ verifies conditions (6), (7) and (8), for almost all $t \in\left[t_{0}, t_{f}\right]$ is called an admissible control for the dynamic system (5). $\mathbb{U}$ denotes the set of admissible controls $Q($.$) .$

Having determined the model (5), the terminal condition (6) and the different constraints (7) and (8), the optimization problem is stated next.

\subsection{Optimization problem}

Let $\mathcal{C}\left(\mathcal{X}\left(t_{f}\right)\right)$ denote the cost function or performance index, namely the FOS concentration at the final time $t_{f}$ defined by

$\mathcal{C}\left(\mathcal{X}\left(t_{f}\right)\right)=\left[\mathrm{GF}_{2}\right]\left(t_{f}\right)+\left[\mathrm{GF}_{3}\right]\left(t_{f}\right)+\left[\mathrm{GF}_{4}\right]\left(t_{f}\right)$.

Notice that $\mathcal{C}\left(\mathcal{X}\left(t_{f}\right)\right)$ is implicitly dependent on the final time $t_{f}$ and the input flow rate $Q(t)$. 
The problem is finally stated as follows:

$$
\begin{aligned}
& \max _{Q \in \mathbb{U}, t_{f} \in \mathbb{R}^{*+}} \mathcal{C}\left(\mathcal{X}\left(t_{f}\right)\right) \\
& \text { subjectto } \frac{d \mathcal{X}(t)}{d t}=f(Q, \mathcal{X})=\mathcal{F}(\mathcal{X})+\mathcal{G}(\mathcal{X}) Q(t) \\
& \mathcal{X}\left(t_{0}\right)=\mathcal{X}_{0} \\
& \psi\left(\mathcal{X}\left(t_{f}\right)\right)=\mathrm{V}\left(t_{f}\right)-\mathrm{V}_{\max } \\
& \mathrm{C}(Q)=\left(\begin{array}{l}
Q_{\min }-Q(t) \\
Q(t)-Q_{\max }
\end{array}\right) \leq 0 \\
& \mathrm{~S}(\mathcal{X})=\left(\begin{array}{c}
\mathrm{X}_{\min }-[\mathrm{X}](t) \\
{[\mathrm{G}](t)-\mathrm{G}_{\max }}
\end{array}\right) \leq 0 .
\end{aligned}
$$

\section{Pontryagin's maximum principle}

This section focuses on how to solve the above mentioned problem. First, penalty terms are added to the performance index to handle the inequality constraints. Next, the Pontryagin's maximum principle is applied. The resulting optimization algorithm is finally described.

\subsection{Regularization and penalty functions}

The maximization problem (9) is a singular control problem because the control variable $Q$ that appears linearly in model (5) is not contained in the performance index $\mathcal{C}\left(\mathcal{X}\left(t_{f}\right)\right)$. In general, a minimum does not exist in this context [28,Chapter 3].

Among the methods found in the literature for dealing with bioprocess optimization and singular control problems, one approach for solving the maximization problem consists in looking for a bangbang control by using the inequality constraints on the control variable. The control function moves only along the boundaries of its constraints. The main idea of this method is then to determine the time instants at which the changes from one extreme value to the other occur. Another method consists in extending the bangbang method by adding singular arcs. The singular control is then defined from the state and costate variables [29,30]. These methodologies are usually used to maximize the amount of cell mass. However, because of the inequality constraints on the byproducts (7) and the complexity of the studied nonlinear system (5), the implementation of such methodologies is not straightforward and may be even inapplicable in our case.

To overcome these difficulties and to ensure the concavity conditions of the function to be maximized, a quadratic integral penalty function depending on the control variable is added to the performance index $\mathcal{C}\left(\mathcal{X}\left(t_{f}\right)\right)$. Let $\mathcal{R}(Q)$ be the term defined by

$\mathcal{R}(Q)=\alpha|Q(t)|^{2}$.

$\alpha$ is a negative constant which determines the importance of the regularization term. The new cost function $J_{0}\left(Q, \mathcal{X}, t_{f}\right)$ to maximize is

$J_{0}\left(Q, \mathcal{X}, t_{f}\right)=\mathcal{C}\left(\mathcal{X}\left(t_{f}\right)\right)+\int_{t_{0}}^{t_{f}} \mathcal{R}(Q) d t$

for which an optimal solution exists to the maximization problem.

A penalty function associated with the inequality constraints is also added to the cost function. It consists of the integral of $\mathcal{P}(Q, \mathcal{X})$ which is the sum of the weighted square of $\mathrm{C}(Q)$ and $\mathrm{S}(\mathcal{X})$

$\mathcal{P}(Q, \mathcal{X})=\mathrm{C}^{\mathrm{T}}(Q) \quad \Omega_{\mathrm{C}} \mathrm{C}(Q)+\mathrm{S}^{\mathrm{T}}(\mathcal{X}) \Omega_{\mathrm{S}} \mathrm{S}(\mathcal{X})$.
The weighting matrices $\Omega_{\mathrm{C} / \mathrm{S}}$ are

$\Omega_{\mathrm{C}}=\operatorname{diag}\left(\begin{array}{lll}\mu_{1}^{\mathrm{C}_{1} \mathrm{C}} & \mu_{2}^{\mathrm{C}_{1}} \mathrm{C}_{2}^{\mathrm{C}}\end{array}\right) \quad$ and $\quad \Omega_{\mathrm{S}}=\operatorname{diag}\left(\begin{array}{lll}\mu_{1}^{\mathrm{S}_{1} \mathrm{~S}} & \mu_{2}^{\mathrm{S}_{1} \mathrm{~S}}{ }_{2}\end{array}\right)$,

where the unit functions $\mathbb{1}$ are defined as

$\mathbb{1}_{i}^{C}=\left\{\begin{array}{cc}0 & \text { if } C_{i}(Q) \leq 0 \\ 1 & \text { otherwise }\end{array}\right.$ for $i=1,2 ;$ and

$\mathbb{1}_{i}^{S}=\left\{\begin{array}{ll}0 & \text { if } S_{i}(\mathcal{X}) \leq 0 \\ 1 & \text { otherwise }\end{array}\right.$ for $i=1,2$

The negative constants $\mu_{i}^{\mathcal{C}}$ and $\mu_{i}^{\mathcal{S}}$ should be specified a priori. Notice that the larger $\left|\mu_{i}\right|$ is, the better the associated constraint is fulfilled [22,28,Chapter 7]. By adjoining the biological, chemical and control inequality constraints to the cost, the corresponding inequality constraints are removed from the initial problem. The cost function to maximize is finally defined by

$J\left(Q, \mathcal{X}, t_{f}\right)=\mathcal{C}\left(\mathcal{X}\left(t_{f}\right)\right)+\int_{t_{0}}^{t_{f}} g(Q, \mathcal{X}) d t$

where $g(Q, \mathcal{X})$ is

$g(Q, \mathcal{X})=\mathcal{R}(Q)+\mathcal{P}(Q, \mathcal{X})$.

When reaching the optimality, the penalty function $\mathcal{P}$ should have a zero contribution to (11) since the inequality constraints should be fulfilled. Hence $J\left(Q, \mathcal{X}, t_{f}\right)=J_{0}\left(Q, \mathcal{X}, t_{f}\right)$ at the optimum. Moreover, let $\tilde{\mathcal{X}}, \tilde{Q}$ and $\tilde{t}_{f}$ respectively be the optimal state trajectory, the optimal input to the system (5) and the final time that maximize the performance index $\mathcal{C}\left(\mathcal{X}\left(t_{f}\right)\right)$ subject to the constraints (7) and (8), and let $\mathcal{X}^{*}, Q^{*}$ and $t_{f}^{*}$ be the same variables as before but that maximize the performance index $J\left(Q, \mathcal{X}, t_{f}\right)$ without constraints. Then, it is reasonable to suppose that $\mathcal{C}\left(\tilde{\mathcal{X}}\left(\tilde{t}_{f}\right)\right) \approx \mathcal{C}\left(\mathcal{X}^{*}\left(t_{f}^{*}\right)\right)$ [28, Chapter 1].

The maximization of $J\left(Q, \mathcal{X}, t_{f}\right)$ is achieved by using Pontryagin's maximum principle, i.e. maximizing the associated Hamilton-Pontryagin function with respect to the control variable, $Q(t)$. First, the main idea of the method is described, next, the algorithm is developed.

\subsection{Optimal control method}

By extending the cost function (11) with the equality constraints defined by the system (5) and the terminal boundary condition (6), and associating each of the latter respectively to a constant multiplier $v$ and a multiplier function $\lambda$, the performance index $J\left(Q, \mathcal{X}, t_{f}\right)^{1}$ to maximize becomes

$J=\mathcal{C}\left(\mathcal{X}\left(t_{f}\right)\right)+v \psi\left(\mathcal{X}\left(t_{f}\right)\right)+\int_{t_{0}}^{t_{f}} g(Q, \mathcal{X})+\lambda^{\mathrm{T}}\{f(Q, \mathcal{X})-\dot{\mathcal{X}}\} d t$

where a part of the integrand is known as the (augmented) Hamilton-Pontryagin function which is symbolized by $H(Q, \mathcal{X})$ and defined as

$H(Q, \mathcal{X})=g(Q, \mathcal{X})+\lambda^{\mathrm{T}} f(\mathcal{X}, Q)$.

A necessary condition for optimality is obtained by imposing the first variation $d J$ to be equal to zero along the optimal control and state trajectories. This leads to the following equation for the costate or adjoint variables (also called influence functions):

$\dot{\lambda}^{\mathrm{T}}=-\lambda^{\mathrm{T}} \frac{\partial f(Q, \mathcal{X})}{\partial \mathcal{X}}-\frac{\partial \mathcal{P}(Q, \mathcal{X})}{\partial \mathcal{X}}$,

\footnotetext{
1 To avoid cumbersome notations, the performance index will be rewritten as $J$ instead of $J\left(Q, \mathcal{X}, t_{f}\right)$.
} 
with the final conditions, also called transversality conditions,

$\lambda^{\mathrm{T}}\left(t_{f}\right)=\left[\frac{\partial \mathcal{C}(Q)}{\partial \mathcal{X}}+v \frac{\partial \psi(\mathcal{X}, t)}{\partial \mathcal{X}}\right]_{t=t_{f}}$

and

$\frac{\partial H(Q, \mathcal{X})}{\partial Q}=\frac{\partial g(Q, \mathcal{X})}{\partial Q}+\lambda^{\mathrm{T}} \frac{\partial f(Q, \mathcal{X})}{\partial Q}=0$.

If $Q$ can be expressed as a function of $\lambda$ and $\mathcal{X}$ from the latter equation, this expression for $Q$ can be substituted in (5), (14) and (15). It yields a two-point boundary-value problem which can be solved for $\mathcal{X}$ and $\lambda$. This problem is quite difficult to solve and an initial solution is required from the users, see [31] and the references therein. Indeed, it was observed that solving model (5) only from a set of terminal conditions leads to numerical instability.

The solution proposed in this paper consists in integrating separately the costate variables backward and the system forward avoiding the instability while estimating the unknown terminal condition of the costate functions. The optimal control input is computed by resorting to a gradient type method, which is now described [28,Chapter 7].

Because one of the state variables is known at the final time, i.e. $\mathrm{V}\left(t_{f}\right)=\mathrm{V}_{\mathrm{max}}$, the transversality conditions contain an undetermined variable $v$. The proper determination of this variable will allow to respect the known final condition in the state variable (6).

\subsection{Algorithm development}

In order to deduce a numerical algorithm to solve the above mentioned problem, the way to account for the known terminal state (Eq. (6)) is reformulated by imposing that changes in $Q$ around the optimal input profile do not affect the fixed state $\mathcal{X}_{8}$ (namely V) at time $t_{f}[28$,Chapter 2]. Starting from (12), without the term $\nu \psi\left(\mathcal{X}\left(t_{f}\right)\right)$ :

$J=\mathcal{C}\left(\mathcal{X}\left(t_{f}\right)\right)+\int_{t_{0}}^{t_{f}} g(Q, \mathcal{X})+\tilde{\lambda}^{\mathrm{T}}\{f(Q, \mathcal{X})-\dot{\mathcal{X}}\} d t$,

and taking the differential while accounting for differential changes in $t_{f}$ yields, after some standard manipulations:

$$
\begin{aligned}
d J & =\left[\frac{\partial \mathcal{C}(\mathcal{X})}{\partial t}+g(Q, \mathcal{X})+\tilde{\lambda}^{\mathrm{T}} f(Q, \mathcal{X})\right]_{t=t_{f}} d t_{f} \\
& +\int_{t_{0}}^{t_{f}}\left[\frac{\partial g(Q, \mathcal{X})}{\partial Q}+\tilde{\lambda}^{\mathrm{T}} \mathcal{G}(\mathcal{X})\right] \delta Q d t
\end{aligned}
$$

where the adjoint variable $\tilde{\lambda}$ are determined in order to eliminate the terms in $\delta \mathcal{X}$ and $\delta \mathcal{X}\left(t_{f}\right)$ that would otherwise appear in (18), namely

$$
\left\{\begin{array}{l}
\dot{\tilde{\lambda}}^{\mathrm{T}}=-\tilde{\lambda}^{\mathrm{T}} \frac{\partial f(Q, \mathcal{X})}{\partial \mathcal{X}}-\frac{\partial \mathcal{P}(Q, \mathcal{X})}{\partial \mathcal{X}}, \\
\tilde{\lambda}\left(t_{f}\right)=\left\{\begin{array}{l}
\left.\frac{\partial \mathcal{C}(\mathcal{X})}{\partial \mathcal{X}_{j}}\right|_{t=t_{f}}, \quad j=1, \ldots, 7 \\
0, j=8 .
\end{array}\right.
\end{array}\right.
$$

Note that in the above expression (18) for $d J,\left.\frac{\partial \mathcal{C}(\mathcal{X})}{\partial t}\right|_{t=t_{f}}=0$ as $\mathcal{C}$ does not depend on $t$ explicitly.

To come up with an algorithm for updating $t_{f}$ and $Q(t), t \in\left[t_{0}\right.$, $t_{f}$, we now consider the change in the final volume $\mathcal{X}_{8}\left(t_{f}\right)$ due to the variations in $\delta Q$, by introducing the influence functions $\hat{\lambda}$. It can be written:

$d \mathcal{X}_{8}\left(t_{f}\right)=\left.f_{8}(Q, \mathcal{X})\right|_{t=t_{f}} d t_{f}+\int_{t_{0}}^{t_{f}} \hat{\lambda}^{\mathrm{T}} \mathcal{G}(\mathcal{X}) \delta Q d t$,

which is equivalent to

$d \mathcal{X}_{8}\left(t_{f}\right)=Q\left(t_{f}\right) d t_{f}+\int_{t_{0}}^{t_{f}} \hat{\lambda}^{\mathrm{T}} \mathcal{G}(\mathcal{X}) \delta Q d t$.

where the effect of $\delta Q$ on $\delta \mathcal{X}$ and $\delta \mathcal{X}_{\mathbf{8}}\left(t_{f}\right)$ is eliminated by setting:

$\left\{\begin{array}{l}\dot{\hat{\lambda}}^{\mathrm{T}}=-\hat{\lambda}^{\mathrm{T}} \frac{\partial f(Q, \mathcal{X})}{\partial \mathcal{X}}, \\ \hat{\lambda}_{j}\left(t_{f}\right)=\left\{\begin{array}{l}0, j=1, \ldots, 7 \\ 1, j=8 .\end{array}\right.\end{array}\right.$

For determining the optimal final time and input trajectory, the algorithm is based on the determination of changes $\delta Q$ and $d t_{f}$ that make $d J>0$ while ensuring $d \mathcal{X}_{8}=0$ in order to fulfill constraint (6). To achieve this goal, let us consider the expression

$d \tilde{J}=d J+v d \mathcal{X}_{8}-\frac{1}{2} \kappa_{t}\left(d t_{f}\right)^{2}$

which is equivalent to

$$
\begin{aligned}
d \tilde{J} & =\left[g(Q, \mathcal{X})+\left(\tilde{\lambda}^{\mathrm{T}}+v \hat{\lambda}^{\mathrm{T}}\right) f(Q, \mathcal{X})-\frac{1}{2} \kappa_{t} d t_{f}\right]_{t=t_{f}} d t_{f} \\
& +\int_{t_{0}}^{t_{f}}\left[\frac{\partial g(Q, \mathcal{X})}{\partial Q}+\left(\tilde{\lambda}^{\mathrm{T}}+v \hat{\lambda}^{\mathrm{T}}\right) \mathcal{G}(\mathcal{X})\right] \delta Q d t .
\end{aligned}
$$

The last term in (22) is a regularization term introduced to ensure the concavity of the problem with respect to $t_{f}$. Note that enforcing $d \tilde{J}>0$ and $d \mathcal{X}_{8}\left(t_{f}\right)=0$ by an appropriate choice of $\delta Q$ and $d t_{f}$ automatically ensures our original requirement $d J>0$. The condition $d \tilde{J}>0$ is obtained with the following selection of $\delta Q$ and $d t_{f}$ :

$\delta Q=\kappa_{Q}^{-1}\left[\frac{\partial g(Q, \mathcal{X})}{\partial Q}+(\tilde{\lambda}+v \hat{\lambda})^{\mathrm{T}} \mathcal{G}(\mathcal{X})\right]$

$d t_{f}=\kappa_{t}^{-1}\left(g(Q, \mathcal{X})+(\tilde{\lambda}+v \hat{\lambda})^{\mathrm{T}} f(Q, \mathcal{X})\right)_{t=t_{f}}$

where $\kappa_{Q}$ and $\kappa_{t}$ are positive constants. Equation (24) leads to an update law for the control trajectory and the final time, namely:

$\hat{Q}:=\hat{Q}+\delta Q$

$\hat{t}_{f}:=\hat{t}_{f}+d t_{f}$

where the denotes an intermediate value of the optimized variable or function like in the algorithm section below (Section 3.5).

On the other hand, $d \mathcal{X}_{8}\left(t_{f}\right)=0$ can be insured by an adequate choice of $v$. The latter is determined by substituting (24a) for $\delta Q$ and (24b) for $d t_{f}$ in (20) and setting the resulting expression to zero. It yields:

$v=-\left(I_{\psi}+R_{1}\right)^{-1}\left(I_{J}+R_{2}\right)$ with

$I_{\psi}=\int_{t_{0}}^{t_{f}} \hat{\lambda}^{\mathrm{T}} \mathcal{G}(\mathcal{X})(\mathcal{G}(\mathcal{X}))^{\mathrm{T}} \quad \hat{\lambda} \quad d t$

$I_{J}=\int_{t_{0}}^{t_{f}} \hat{\lambda}^{\mathrm{T}} \mathcal{G}(\mathcal{X})\left[\frac{\partial g(Q, \mathcal{X})}{\partial Q}+\tilde{\lambda}^{\mathrm{T}} \mathcal{G}(\mathcal{X})\right] d t$,

$R_{1}=\frac{\kappa_{Q}}{\kappa_{t}}\left(Q\left(t_{f}\right)\right)^{2}$,

$R_{2}=\frac{\kappa_{Q}}{\kappa_{t}} Q\left(t_{f}\right)\left(g(Q, \mathcal{X})+\tilde{\lambda}^{\mathrm{T}} f(Q, \mathcal{X})\right)_{t=t_{f}}$. 
In order to determine the stopping criterion of an algorithm based on the update law (25), notice that, when the solution associated to the maximum of $J$ is achieved, $\delta Q$ and $d t_{f}$ tend to zero. Hence,

$$
\begin{aligned}
& {\left[\frac{\partial g(Q, \mathcal{X})}{\partial Q}+(\tilde{\lambda}+v \hat{\lambda})^{\mathrm{T}} \mathcal{G}(\mathcal{X})\right] \rightarrow 0 \text { forall } t \in\left[t_{0}, t_{f}\right]} \\
& \left(g(Q, \mathcal{X})+(\tilde{\lambda}+v \hat{\lambda})^{\mathrm{T}} f(Q, \mathcal{X})\right)_{t=t_{f}} \rightarrow 0
\end{aligned}
$$

Expression (27a) can be expressed in terms of the Hamilton-Pontryagin function defined in (13) which can be rewritten

$H(Q, \mathcal{X}) \equiv g(Q, \mathcal{X})+(\tilde{\lambda}+v \hat{\lambda}) f(Q, \mathcal{X})$,

Indeed, (27a) is equivalent to $\frac{\partial H}{\partial Q}=0$. Besides the influence functions characterized by (19) and (21) allow a decomposition of the initial influence functions defined in Eqs. (14) and (15) as

$\lambda=\tilde{\lambda}+v \hat{\lambda}$.

Finally notice that constraint (6) must also be fulfilled for the optimal solution and it will thus be included in the stopping criterion of the algorithm.

\subsection{Analysis of the trajectory of the optimal substrate feed rate}

Let us first summarized the necessary optimality conditions

$(g(Q, \mathcal{X})+(\tilde{\lambda}+v \hat{\lambda}) f(Q, \mathcal{X}))_{t=t_{f}}=0$

$\frac{\partial g(Q, \mathcal{X})}{\partial Q}+(\tilde{\lambda}+\nu \hat{\lambda})^{\mathrm{T}} \mathcal{G}(\mathcal{X})=0$, forall $t \in\left[t_{0}, t_{f}\right]$

$\psi\left(\mathcal{X}\left(t_{f}\right)\right)=\mathrm{V}\left(t_{f}\right)-\mathrm{V}_{\max }=0$.

From (29b), where the control variable appears explicitly, the optimal trajectory for $Q$, denoted $Q^{*}$, is determined as

$Q^{*}=\frac{\lambda^{* \mathrm{~T}} \mathcal{G}\left(\mathcal{X}^{*}\right)-2 \mu_{1}^{\mathrm{C}} Q_{\min } 1_{1}^{\mathrm{C}}-2 \mu_{2}^{\mathrm{C}} Q_{\max } 1_{2}^{\mathrm{C}}}{-2 \alpha-2 \mu_{1}^{\mathrm{C}} \mathbb{1}_{1}^{\mathrm{C}}-2 \mu_{2}^{\mathrm{C}} \mathbb{1}_{2}^{\mathrm{C}}}$

in terms of the optimal values for $\lambda$ and $\mathcal{X}$, namely $\lambda^{*}$ and $\mathcal{X}^{*}$. The obtained $\hat{Q}$ in (25a) should converge to $Q^{*}$, upon termination of the algorithm. Since, the control variable does not appear directly in the state constraints, these constraints are not explicitly present in expression (30). However, they are indirectly taken into account through the influence functions $\lambda$. Nevertheless, as long as [G] (respectively $[\mathrm{X}]$ ) follows the boundaries of its constraint, which is linear in the state, the first-order time derivative of its constraint should be constant or negative (respectively positive). This implies

$$
\begin{aligned}
& \text { if } \quad[G](t)-G_{\max } \geq 0 \quad \text { then } \quad \frac{d[G](t)}{d t} \leq 0, \\
& \text { if }-[\mathrm{X}](t)+\mathrm{X}_{\min } \geq 0 \text { then } \frac{d[\mathrm{X}](t)}{d t} \geq 0 .
\end{aligned}
$$

Since the temporal dynamics of $[G]$ and $[X]$ are function of the feed flow (Eq. (5)), the control variable has to respect, according to Eqs. (31) and (32), the following constraints:

$Q(t) \geq \frac{V}{[\mathrm{G}]}\left(k_{1} r_{1}+\frac{k_{10} r_{5}}{2}-\mathrm{Y}_{\mathrm{G}} r_{8}\right) \mathbb{1}_{2}^{\mathrm{S}}$,

and

$Q(t) \leq \frac{V}{[\mathrm{X}]}\left(r_{8}+r_{9}\right) 1_{1}^{S}$,

assuming $[G] \neq 0$ and $[X] \neq 0$.

It has to be noticed that due to the inequality constraints on the biomass, the glucose and the substrate feed rate, incompatibility on the constraints can occur and may create infeasibility of the input trajectory. It is obvious that no optimal solution will exist if one of the following situations happens

$$
\begin{aligned}
& \frac{V}{[\mathrm{X}]}\left(r_{8}+r_{9}\right) \mathbb{1}_{1}^{S}>Q_{\max } ; \\
& \frac{V}{[\mathrm{G}]}\left(k_{1} r_{1}+\frac{k_{10} r_{5}}{2}-\mathrm{Y}_{\mathrm{G}} r_{8}\right) \mathbb{1}_{2}^{S}>Q_{\max } ; \\
& \frac{V}{[\mathrm{X}]}\left(r_{8}+r_{9}\right) \mathbb{1}_{1}^{S}<Q_{\min } ; \\
& \frac{V}{[\mathrm{G}]}\left(k_{1} r_{1}+\frac{k_{10} r_{5}}{2}-\mathrm{Y}_{\mathrm{G}} r_{8}\right) \mathbb{1}_{2}^{S}<Q_{\min } ; \\
& \frac{V}{[\mathrm{X}]}\left(r_{8}+r_{9}\right) \mathbb{1}_{1}^{S}<\frac{V}{[\mathrm{G}]}\left(k_{1} r_{1}+\frac{k_{10} r_{5}}{2}-\mathrm{Y}_{\mathrm{G}} r_{8}\right) \mathbb{1}_{2}^{S} .
\end{aligned}
$$

When the optimality conditions are reached, the resulting control input and final time should be equivalent to the optimal input and optimal final time respectively, i.e. $\hat{Q} \equiv Q^{*}$ and $\hat{t}_{f} \equiv t_{f}^{*}$, and the inequalities defined in (35a)-(35e) should not hold when $\hat{Q} \equiv Q^{*}$ and $\hat{t}_{f} \equiv t_{f}^{*}$.

\subsection{Algorithms}

An algorithm for estimating $\delta Q$ and $d t_{f}$ in order to generate a solution that fulfills the above conditions consists in the following steps:

\section{Algorithm I}

1 Guess a final time $\hat{t}_{f}$ and an initial input function $\hat{Q}(t), t \in\left[t_{0}, \hat{t}_{f}\right]$

2 Solve forward the system (5) with the initial condition $\mathcal{X}_{0}$.

3 Integrate backward Eqs. (19) and (21) with their indicated final conditions to determine the costate functions $\tilde{\lambda}$ and $\hat{\lambda}$.

4 Determine $v$ from (26) and $\delta \hat{Q}$ and $d \hat{t}_{f}$ from (24a) and (24b), respectively.

5 Assign $\hat{Q}:=\hat{Q}+\delta \hat{Q}, \hat{t}_{f}:=\hat{t}_{f}+d \hat{t}_{f}$.

6 Repeat steps (2) through (5) until the optimality conditions, defined by (29a)-(29c), are satisfied.

Fulfilling the inequality conditions (7) and (8) in the initialization step is not necessary for the algorithm convergence, given the penalty function approach used for handling constraints. The implemented algorithm is based on the previous development involving the decomposition of the costate variables. Because the paper focuses on the FOS concentration optimization (chemical aspects and optimization), all the theoretical aspects are not shown here, and the readers can refer to [28] or [21] for more explanation. Nevertheless, in the following subsections, the selection of the different algorithm parameters is discussed, namely the scheduling parameters $\kappa_{Q}$ and $\kappa_{t}$, the regularization parameter $\alpha$ and the penalty function parameter $\mu$.

\subsubsection{Selection of the scheduling parameters $\kappa$}

The convergence speed of the algorithm depends of the selection of the $\kappa_{i}$-parameters (for $i=Q, t$ ). While good selection of these parameters may improve the direction of the path to the optimal solution, on the contrary the algorithm may diverge for poorly chosen values [28]. To overcome this selection issue, two different approaches may be used in the optimization problem: (i) the linesearch approach based on the adjustment of the steplength $\kappa_{i}$ in a specific direction [32], (ii) the trust-region based method which focuses on the optimization of a second cost function with respect to the search direction [33,34].

The line-search method is considered here. This method requires to add three steps within the initial Algorithm I 
1 Select initial values for $\kappa_{Q}$ and $\kappa_{t}$

2 Compute $J\left(\hat{Q}+\delta \hat{Q}\left(\kappa_{Q}\right), \hat{t}_{f}+d \hat{t}_{f}\left(\kappa_{t}\right)\right)-J\left(\hat{Q}, \hat{t}_{f}\right)$. If the latter is sufficiently large then $\hat{Q}:=\hat{Q}+\delta \hat{Q}, \hat{t}_{f}:=\hat{t}_{f}+d \hat{t}_{f}$.

3 Otherwise, $\kappa_{i}$ is reduced such as $\kappa_{i}:=\rho_{i} \kappa_{i}$, with $\left.\rho_{i} \in\right] 0,1$ [for $i=Q$, $t$.

More details on the theoretical aspects and on the convergence conditions can be found in [21]. Taking into consideration these new steps, the algorithm is now:

Algorithm II

1 Guess an initial control input $\hat{Q}$ and a final time $\hat{t}_{f}$.

2 Select initial values for $\kappa_{Q}$ and $\kappa_{t}$.

3 Solve forward the system (5) with the initial condition $\mathcal{X}_{0}$.

4 Integrate backward Eqs. (19) and (21) with their indicated final conditions to determine the costate functions $\tilde{\lambda}$ and $\hat{\lambda}$.

5 Determine $v$ from (26) and $\delta \hat{Q}$ and $d \hat{t}_{f}$ from (24a) and (24b), respectively.

6 Compute $J\left(\hat{Q}+\delta \hat{Q}\left(\kappa_{Q}\right), \hat{t}_{f}+d \hat{t}_{f}\left(\kappa_{t}\right)\right)-J\left(\hat{Q}, \hat{t}_{f}\right)$. If the latter is sufficiently large then $\hat{Q}:=\hat{Q}+\delta \hat{Q}, \hat{t}_{f}:=\hat{t}_{f}+d \hat{t}_{f}$. Otherwise, $\kappa_{i}$ is reduced such as $\kappa_{i}:=\rho_{i} \kappa_{i}$, with $\left.\rho_{i} \in\right] 0,1[$ for $i=Q$, , go back step 3

7 Repeat steps (2) through (6) until the optimality conditions, defined by (29a)-(29c), are satisfied.

\subsubsection{Tuning of the regularization parameter $\alpha$}

As previously explained, the regularization term given by $\mathcal{R}$ allows to solve the singular control problem. However, a wrong choice of the associated parameter $\alpha$ may change the accuracy of the control profile, whereas the solution for the initial singular control problem (9) should theoretically be obtained with an $\alpha=0$ in (11). To overcome this issue, the main idea found in the literature is to consider the penalty parameter $\alpha$ as a decreasing sequence (see for example [21, Chapter 10] and the reference therein or [35]). The decline of the parameter would end before reaching numerical instability while tending to the singular solution. Algorithm II is then augmented as follows, with $\alpha:=\alpha_{k}$ :

Algorithm III

1 Selection of a starting value $\alpha_{k}$ and $k=0$.

2 Run algorithm II.

3 Chose $\alpha_{k+1}=\rho_{\alpha} \alpha_{k}$ with $\rho_{\alpha} \in$ ]0, 1 [and $k=k+1$, repeat step 2

4 Algorithm III stops when numerical instability in Algorithm II occurs because the value of $\alpha$ is too small. This is done by including a stopping criterion such as: $\left\|Q_{k+1}-Q_{k}\right\|_{2}>\epsilon_{Q}$ where $\epsilon_{Q}$ is a user defined parameter and $\|\cdot\|_{2}$ stands for the $\mathrm{L}_{2}$ norm of the signal over the interval $\left[0, t_{f}\right]$.

Remark: The developed algorithms are based on the first-order gradient method which may be very slow to converge to the optimum. The first run of Algorithm II (when embedded in algorithm III) may indeed take several hundred iterations before converging to the optimal solution.
3.5.3. Tuning of the penalty function parameters $\mu_{\mathrm{i}}$

Concerning the parameters $\mu_{i}^{\mathrm{S}}$ and $\mu_{i}^{\mathrm{C}}$, their value should be as large as possible in order to satisfy the constraints. Nevertheless, high values of these parameters may create a narrow valley containing the optimal solution and may render the numerical computation difficult [28,Chapter 1]. To obtain a suitable compromise, Algorithm III is run successively for different values of $\mu_{i}^{\mathrm{S}}$ and $\mu_{i}^{\mathrm{C}}$. If the $i$ th state inequality constraint is fulfilled, $\mu_{i}^{\mathrm{S}}$ is updated as $\mu_{i}^{\mathrm{S}}:=\mu_{i}^{\mathrm{S}} \times \delta_{\mu}$ where $\delta_{\mu}<1$ at the next algorithm execution; otherwise $\mu_{i}^{\mathrm{S}}:=\mu_{i}^{\mathrm{S}} / \delta_{\mu}$. The size of $\delta_{\mu}$ depends on the amount of constraint violation. A similar update method is used for $\mu_{i}^{\mathrm{C}}$. This procedure ensures a sufficient slope of the valley around the optimum.

\section{Results and discussion}

In this section, the proposed algorithm is used for batch and fed-batch control to optimize the FOS concentration $\mathcal{C}\left(\mathcal{X}\left(t_{f}\right)\right)$ at the undetermined final time $t_{f}$. Two main scenarios are considered. First the batch case is studied, next three fed-batch designs are investigated, and the results are discussed.

\subsection{Operating conditions}

The parameters used in the process model are referenced in Tables 1 and 2 .

These parameters were obtained for a $\mathrm{pH}$ of 5 and a temperature of $30^{\circ} \mathrm{C}$ which correspond to the conditions for the fermentative production of FOS with Aspergillus sp. through transfructosylating enzymes used in [5]. The initial conditions for batch and fed-batch differ but the total sucrose quantity is the same for both cases. These conditions are fixed and are not included in the optimization problem. Keeping the same sucrose quantity will allow a fair comparison between both situations.

Concerning the algorithm, we consider that the optimality is reached when the scalars in the left hand side of (29a) and (29c) are lower than $10^{-8}$ and the $\mathrm{L}_{2}$ norm of the partial derivative of the Hamilton-Pontryagin function with respect the variable $Q \frac{\partial H}{\partial Q}$, (29b) is lower than $10^{-5}$.

\subsection{Results for the batch case}

The objective of this first case is to determine the unspecified final time instant $t_{f}$ that maximizes the concentration $\mathcal{C}$ of FOS. In this situation, $Q$ is set to zero. This case can be seen as a check of the digital implementation of the proposed algorithm since the unspecified final time value can be easily determined by numerically solving the system (5) for a large time scale. Considering an initial sucrose concentration of $333.3 \mathrm{~g} \mathrm{~L}^{-1}$ (Table 3), the maximal FOS concentration was found at the final time, $\hat{t}_{f, \text { batch }}=28 \mathrm{~h}$. FOS maximal concentration, productivity and yield obtained were, respectively, $\mathcal{C}\left(\hat{t}_{f, \text { batch }}\right)=191.9 \mathrm{gL}^{-1}, \mathcal{P}_{\text {rod }}\left(\hat{t}_{f, \text { batch }}\right)=$

Table 2

Simulation parameters.

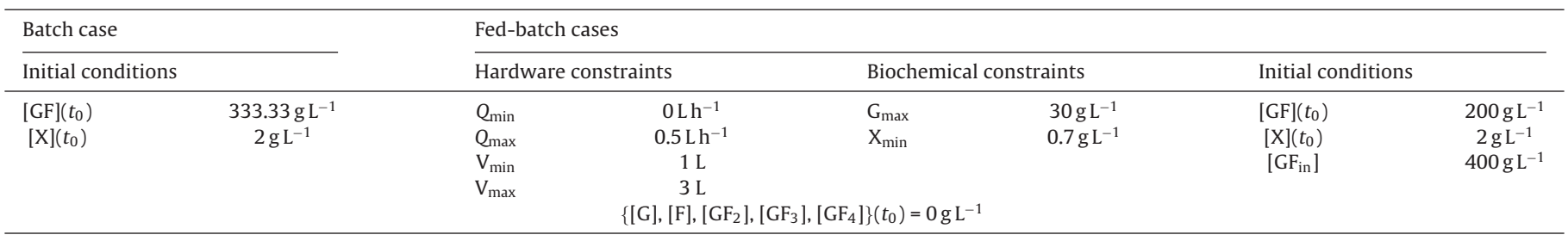


Table 3

Results of the batch and fed-batch optimization procedure.

\begin{tabular}{lcccc}
\hline & Batch & Fed-batch 1 & Fed-batch 2 & Fed-batch 3 \\
\hline $\mathcal{C}\left(t_{f}\right)\left(\mathrm{g}_{\text {FOS L }}-1\right)$ & 191.9 & 192.5 & 191.9 & 72.4 \\
$\mathcal{Y}\left(t_{f}\right)(\%)$ & 61.78 & 61.83 & 61.71 & 67.54 \\
$\mathcal{P}_{\text {rod }}\left(t_{f}\right)\left(\mathrm{g}_{\mathrm{FOS} \mathrm{L}}{ }^{-1} \mathrm{~h}^{-1}\right)$ & 6.8 & 6.31 & 6.5 & 7.46 \\
$t_{f}(\mathrm{~h})$ & 28.1 & 31.2 & 32 & 9.7 \\
\hline
\end{tabular}

$6.8 \mathrm{~g} \mathrm{~L}^{-1} \mathrm{~h}^{-1}$ and $\mathcal{Y}\left(\hat{t}_{f, \text { batch }}\right)=61.78 \%$. The latter two are given by the following equations [36]

$\mathcal{P}_{\text {rod }}\left(t_{f}\right)=\frac{\left[\mathrm{GF}_{2}\right]\left(t_{f}\right)+\left[\mathrm{GF}_{3}\right]\left(t_{f}\right)+\left[\mathrm{GF}_{4}\right]\left(t_{f}\right)}{t_{f}}$.

$\mathcal{Y}\left(t_{f}\right)=\frac{\left[\mathrm{GF}_{2}\right]\left(t_{f}\right)+\left[\mathrm{GF}_{3}\right]\left(t_{f}\right)+\left[\mathrm{GF}_{4}\right]\left(t_{f}\right)}{[\mathrm{GF}]\left(t_{0}\right)-[\mathrm{GF}]\left(t_{f}\right)}$

In the present work, a high sucrose concentration $\left(333.33 \mathrm{~g} \mathrm{~L}^{-1}\right)$ was used in the batch fermentation. The considered concentration is higher than the one used in previous reports where FOS were experimentally produced in batch cultures with Aspergillus sp., namely around $200 \mathrm{~g} \mathrm{~L}^{-1}$ [37-39].

The high concentration of sucrose in the broth leads to higher production of FOS. It was reported that both FTase concentration and specific enzyme activity increase with increasing sucrose concentration up to $280 \mathrm{~g} \mathrm{~L}^{-1}[40,41]$. However, when the concentration of this substrate in the medium is too high, an osmotic stress can be induced in the cells, which can reduce the cells growth and thus inhibit the transfructosylating reactions. Thus, before setting the maximal sucrose concentration in the broth, previous experimental tests should be performed using the specific working microorganism. An estimation of this value was used in the present work.

To allow the use of high sucrose concentration without affecting cells performance, fed-batch fermentation mode can be used. Maximal enzyme production in fed-batch cultures was reported to be about 20\% higher than that in batch cultures [40]. This effect may be due to both the reduction of substrate inhibition inducing osmotic stress in the cells, which promotes cell growth, and the diluting effect in sucrose-feeding operations, which reduces broth viscosity and hence increases mass transfer efficiency. As a result, better cellular metabolic and enzyme synthesizing activities could be maintained leading to higher FOS production rate [40]. On the other hand, at low or residual sucrose concentration, the substrate is rapidly hydrolyzed by the hydrolytic enzymes to glucose and fructose for cells growth, rather than used by the transfructosylating enzymes for FOS synthesis [39].

\subsection{Results for the fed-batch case}

Consider now the fed-batch case. The final time $t_{f}$ and the feed stream $Q$ are determined in order to meet the biochemical (7), control (8) and terminal constrains (6) and maximize the FOS concentration. The optimization control problem is solved for three different cases, namely:

1 Fed-batch 1: Only hardware constraints (8) are taken into account, no biochemical constraint is considered.

2 Fed-batch 2: It extends fed-batch 1 by considering the biochemical constraint (7) on the biomass, i.e. $\mu_{1}^{S} \neq 0$ and $\mu_{2}^{S}=0$ in Eq. (10).

3 Fed-batch 3: It extends fed-batch 2 by considering the biochemical constraint on the glucose concentration, i.e. $\mu_{1}^{S} \neq 0$ and $\mu_{2}^{S} \neq 0$ in Eq. (10).
The results are given in Table 3 where the yield $\mathcal{Y}\left(t_{f}\right)$ is now defined by [36]

$Y\left(t_{f}\right)=\frac{\mathrm{V}_{\max }\left(\left[\mathrm{GF}_{2}\right]\left(t_{f}\right)+\left[\mathrm{GF}_{3}\right]\left(t_{f}\right)+\left[\mathrm{GF}_{4}\right]\left(t_{f}\right)\right)}{[\mathrm{GF}]\left(t_{0}\right) \mathrm{V}\left(t_{0}\right)+\left[\mathrm{GF}_{\mathrm{in}}\right]\left(\mathrm{V}_{\max }-\mathrm{V}\left(t_{0}\right)\right)-[\mathrm{GF}]\left(t_{f}\right) \mathrm{V}_{\max }}$.

In the remainder of this section, the initialization of $Q$ and $t_{f}$ and the tuning parameters for Algorithm II and Algorithm III are shown, followed by a successive presentation of the results obtained for each fed-batch case.

\subsubsection{Initialization of Algorithm II}

The initialization step for algorithm II can be performed as follows for the different cases. Note that the initial choice of the input profile $\hat{Q}(t), t \in\left[t_{0}, \hat{t}_{f}\right]$ need not provide a feasible state trajectory as the penalty function approach will enforce such constraints throughout the run of the algorithm.

1 Fed-batch 1: The final time $\hat{t}_{f}$ is initialized at the optimal final time estimated for the batch case, $t_{f, b a t c h}^{*}$. The bang-bang method is used to find a feasible solution $\hat{Q}_{1}(t), t \in\left[t_{0}, t_{f, b a t c h}^{*}\right]$.

2 Fed-batch 2: A feasible solution is not straightforward to find in this situation. Accepting a violation of the constraint on the biomass in the first iterations, the optimal final time $t_{f 1}^{*}$ and the optimal input profile obtained in fed-batch 1 (denoted $\hat{Q}_{1}^{*}(t), t \in$ $\left.\left[t_{0}, t_{f 1}^{*}\right]\right)$ may then be used, i.e. $\hat{Q}_{2}(t)=\hat{Q}_{1}^{*}(t), t \in\left[t_{0}, t_{f 1}^{*}\right]$.

3 Fed-batch 3: The optimal substrate feed rate obtained in fedbatch 2 (denoted $\hat{Q}_{2}^{*}(t), t \in\left[t_{0}, t_{f 2}^{*}\right]$ ) is employed to initialize this case. However, to avoid a strong violation of the constraint on the glucose concentration the following change was performed. The final time was decreased to minimize the violation of the biochemical constraints. This has induced a modification of the vessel final volume $V\left(t_{f}\right)$, see Eq. (6), i.e. $\psi\left(\mathcal{X}\left(t_{f}\right)\right) \neq 0$ during the first iterations of the algorithm. Thus $\hat{Q}_{3}(t)=\hat{Q}_{2}^{*}(t), t \in\left[t_{0}, \hat{t}_{f}\right]$ where $\hat{t}_{f}<t_{f 2}^{*}$.

\subsubsection{Parameter selection for Algorithm III}

The following initial value of $\alpha$ was used $\alpha=1$. The values of the scheduling parameters were $\kappa_{Q}=1.10^{15}$ and $\kappa_{t}=1.10^{8}$. The multiplicative factors were chosen as: $\rho_{\alpha}=0.99, \rho_{Q}=0.999$ and $\rho_{t}=0.99$. $\mu_{i}^{\mathrm{C}}$ are chosen to ensure that the feed flow constraints are fulfilled up to a small excess, i.e. $0.001 \mathrm{Lh}^{-1}$. A $5 \%$ maximum excess on the state constraints is acceptable, enabling small values of $\mu_{i}^{\mathrm{S}}$. Initially, the weigthting coefficients for the penalty functions were $\mu_{i}^{j}=-1.10^{5}$ for $j=S, C$ and $i=1,2$.

\subsubsection{Fed-batch 1}

This first case focuses on the determination of an admissible control variable to achieve the maximal FOS concentration with constraints only on the control variable. The optimality is reached with respect to the criteria defined above by Eqs. (29a)-(29c). In the upper plot of Fig. 1, the optimal substrate feed rate profile $\hat{Q}_{1}^{*}$ is displayed, which is divided into two phases. First, the bioreactor is filled, next it operates in a batch mode where no substrate feed is added, i.e. $Q_{\min }=0 \mathrm{Lh}^{-1}$, letting the enzymatic reactions to occur.

The fit quality between the value of $Q_{1}^{*}$ deduced from (30) and the obtained input $\hat{Q}_{1}^{*}$ is computed from the following equation

$\mathrm{F}_{\mathrm{it}}=100\left(1-\frac{\int_{0}^{t_{f}}\left(\hat{\mathrm{Q}}-Q^{*}\right)^{2} d t}{\int_{0}^{t_{f}} \hat{Q}^{2} d t}\right)$.

In this case, $\mathrm{F}_{\mathrm{it}}$ is $99.8 \%$. The $0.2 \%$ mismatch is due to numerical errors given that $\frac{\partial H}{\partial Q}$ is not completely zero. Similar results for the $F_{\text {it }}$ are obtained in the next two fed-batch scenarios. 

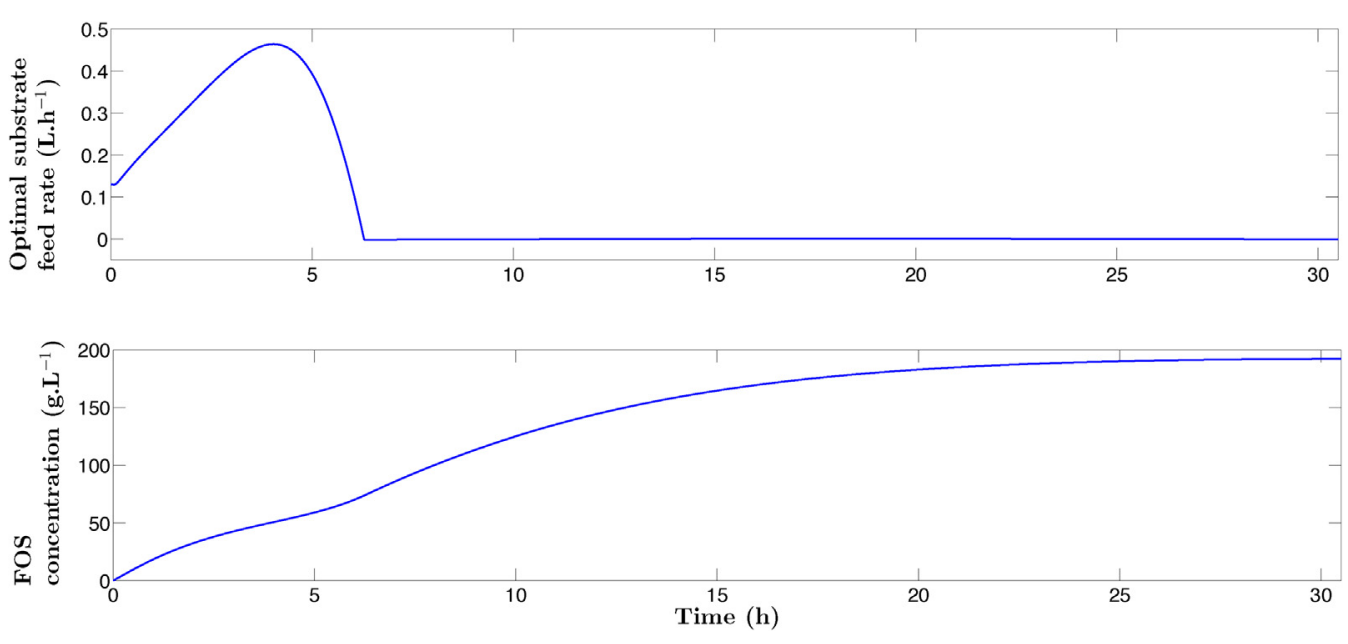

Fig. 1. Case fed-batch 1 . Upper: Optimal substrate feed rate $\hat{Q}_{1}^{*}$. Lower: Time evolution of the FOS concentration.

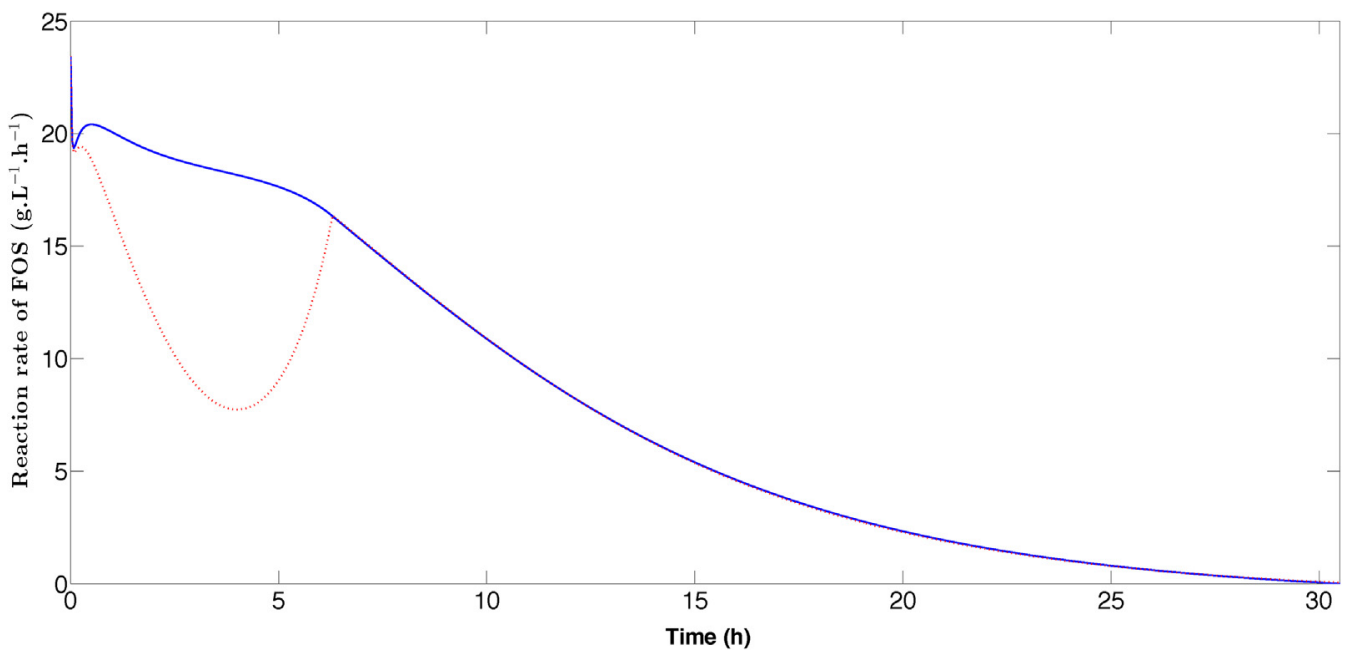

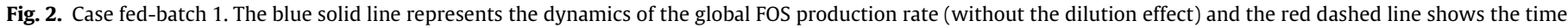

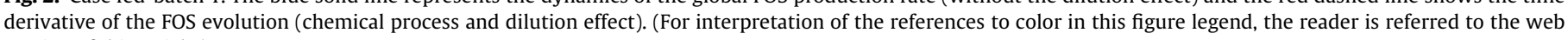
version of this article.)

From a chemical point of view, feeding the bioreactor from the beginning limits the decline of dynamics of the global FOS production rate, which can be computed by the sum $\mathcal{F}_{\mathrm{GF}_{2}}+\mathcal{F}_{\mathrm{GF}_{3}}+\mathcal{F}_{\mathrm{GF}_{4}}{ }^{2}$ (cf. Eq. (5)). The latter is represented by the blue solid line in Fig. 2. However, accounting for the dilution effects lowers these dynamics in the presence of any feeding rate $\left(f_{\mathrm{GF}_{2}}+f_{\mathrm{GF}_{3}}+f_{\mathrm{GF}_{4}}\right)$, while returning to the initial scenario without dilution once the feeding is over (red dashed line in Fig. 2). This case shows the influence of the dilution effects on the reaction rates. Since the dilution part (represented by $\mathcal{G}$ ) is higher than the main dynamics (represented by $\mathcal{F}$ ), the FOS concentration decreases considerably during the feeding. It follows that the fed-batch situation may not be satisfying for improving the production of FOS. Moreover, the obtained final concentration of FOS is in this case equivalent to the batch case. However, as explained in fed-batch 2 and 3 below, fed-batch scenarios allow to control the different reaction rates together with by-product concentrations.

Remark: In practice, feeding the bioreactor with a non-linear feed rate kinetics of sucrose as the one shown in Fig. 1 requires a

\footnotetext{
${ }^{2}$ The subscript indicates which component of the associated function is considered.
}

feasible approximation of the profile throughout the fermentation process. This can be performed using digitally controlled peristaltic pumps, known for their high precision, that can accurately feed the bioreactor throughout the fermentation time.

\subsubsection{Fed-batch 2}

This case comprises the fed-batch 1 case plus the biochemical constraint on the biomass. Three feeding phases can be distinguished in this fed-batch scenario as indicated by the vertical black dotted lines in the upper plot of Fig. 3. In the first phase the feed profile is quite similar to fed-batch 1 . Around the time instant $t=6.1 \mathrm{~h}$, the feed rate is modified in order to avoid the biomass concentration to decrease below $\mathrm{X}_{\min }=0.7 \mathrm{~g} \mathrm{~L}^{-1}$ (see middle plot of Fig. 3), which is the starting point of phase 2 . The optimal control input computed in phase 2 exhibits a magnitude considerably smaller when compared with that of phase 1 . This aspect promotes the limitation of the dilution effects during phase 2 (characterized by $\mathcal{G}_{\mathrm{X}}$ ) as represented by the blue line in Fig. 4 . Such a limitation stimulates the chemical reaction rate associated to the biomass (characterized by $\mathcal{F}_{\mathrm{X}}$ in Eq. (5)), as exhibited by its increase during phase 2 (red line in Fig. 4). After phase 2, the control input goes to $Q_{\min }=0 \mathrm{~L} \mathrm{~h}^{-1}$, that is when the reactor performs in batch mode. This situation marks the beginning of phase 3 . 


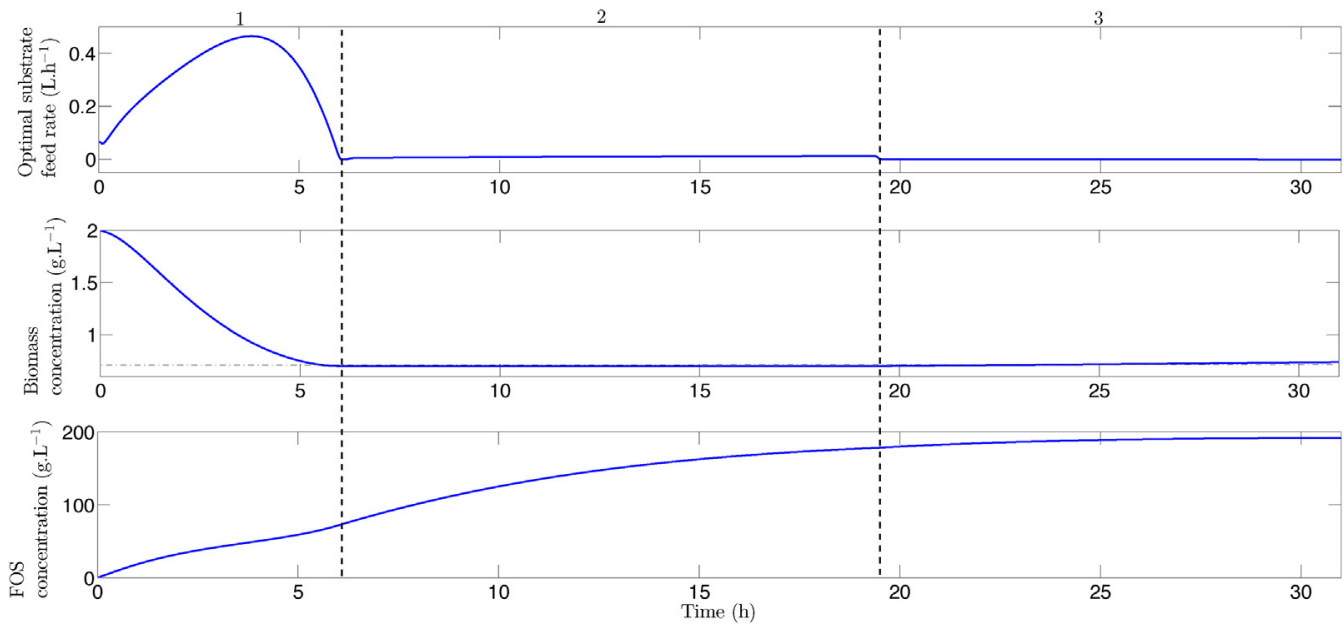

Fig. 3. Case fed-batch 2. Upper: Optimal substrate feed rate $\hat{Q}_{2}^{*}$. Middle: Time evolution of the biomass. Lower: Time evolution of the FOS concentration.

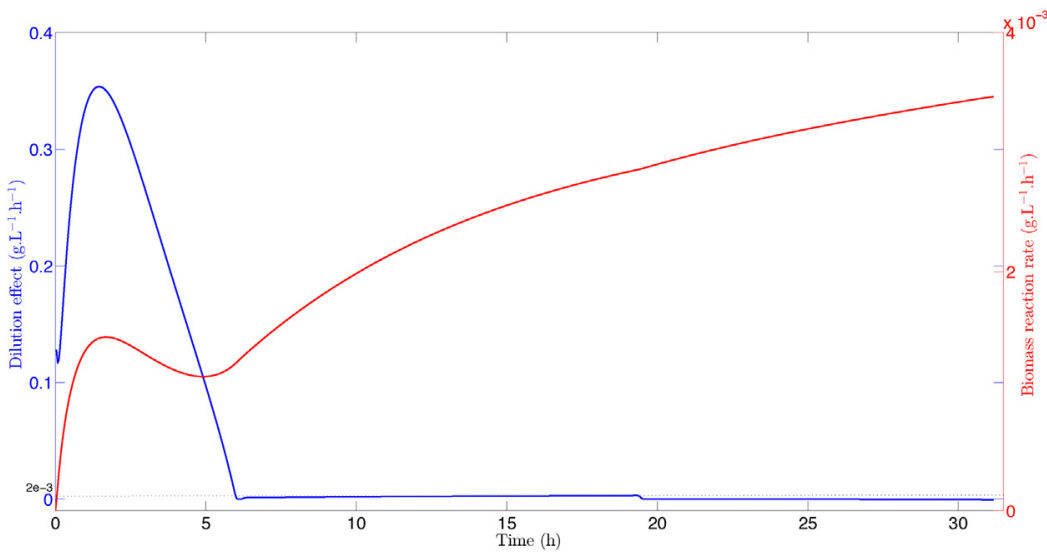

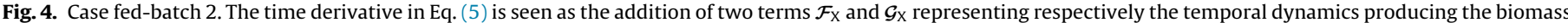

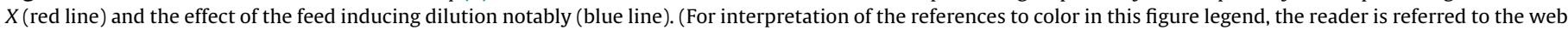
version of this article.)

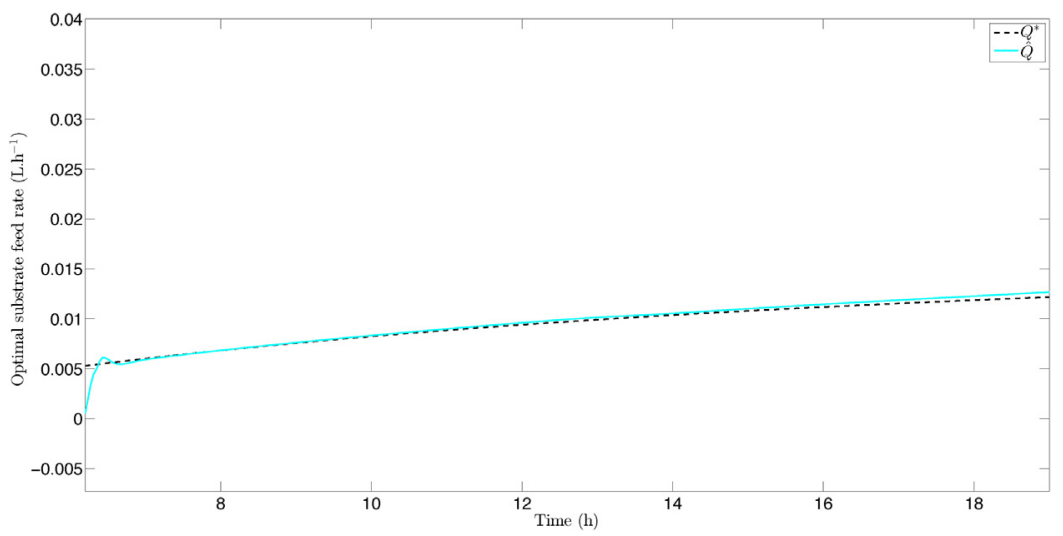

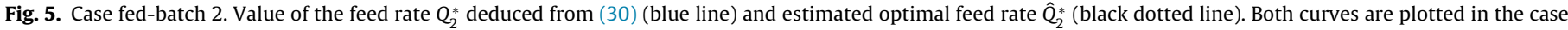

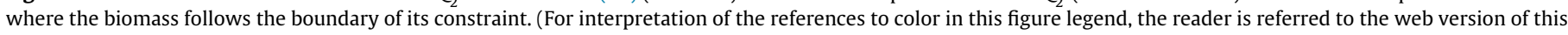
article.)

At $\hat{t}_{f 2} \approx 32 \mathrm{~h}$, the maximal FOS concentration is achieved and corresponds to $\mathcal{C}\left(\hat{t}_{f 2}\right) \approx 191.9 \mathrm{gL}^{-1}$ (see lower plot of Fig. 3 ). These results are similar to the fed-batch 1 case, although in this situation, the biomass concentration is always higher than the minimal value of $0.7 \mathrm{~g} \mathrm{~L}^{-1}$ thanks to the substrate feed rate control.
As previously seen, during the second phase the biomass concentration rides its constraint, which implies that the control function can be determined by (34). Fig. 5 shows $Q_{2}^{*}$ computed from

$$
Q_{2}^{*}(t)=\frac{V}{[\mathrm{X}]}\left(r_{8}+r_{9}\right) \mathbb{1}_{1}^{\mathrm{S}},
$$




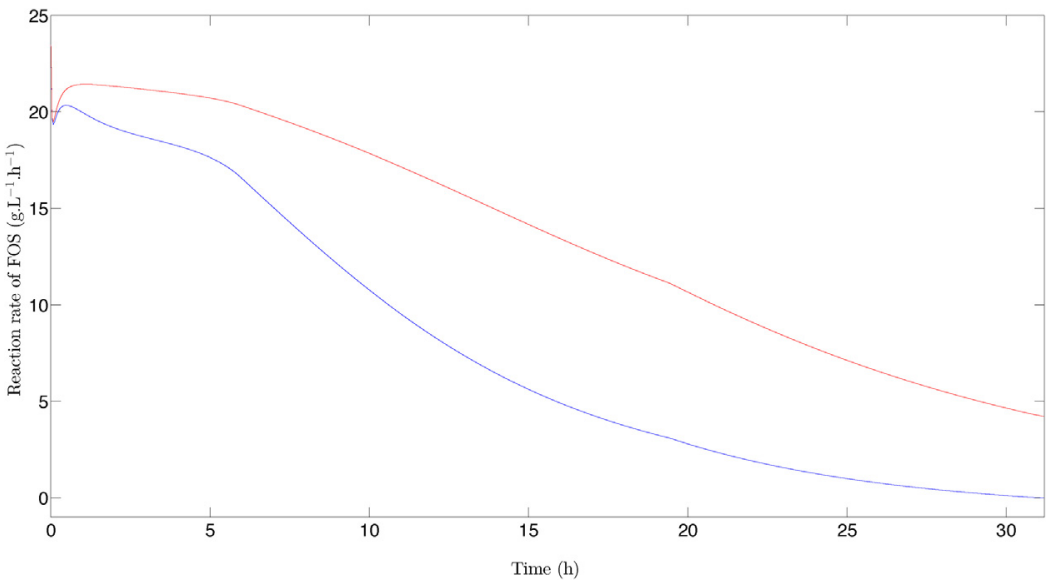

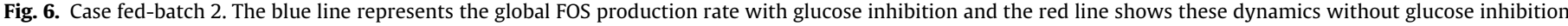

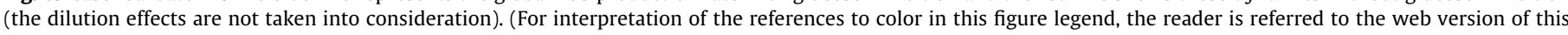
article.)

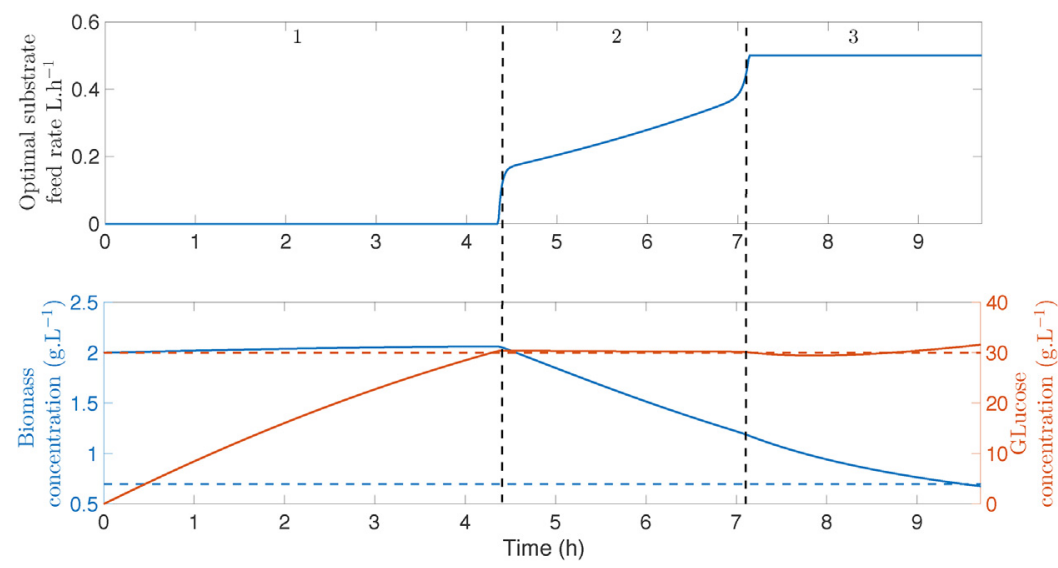

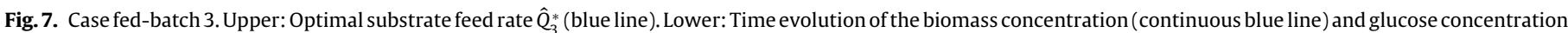

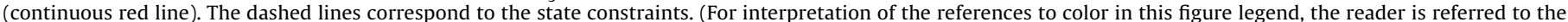
web version of this article.)

which is equivalent to Eq. (30), along with the obtained control input $\hat{Q}_{2}^{*}$. As expected, both are similar.

Besides the dilution effect discussed above, another effect that decreases the dynamics of the FOS chemical process concerns the inhibition promoted by the presence of glucose in the reaction. The global FOS production rate given by $\mathcal{F}_{\mathrm{GF}_{2}}+\mathcal{F}_{\mathrm{GF}_{3}}+\mathcal{F}_{\mathrm{GF}_{4}}$ is shown in Fig. 6, where the red line represents the scenario without inhibition, i.e. the three ratios $\frac{[G]}{\mathrm{Kgst}}, \frac{[\mathrm{G}]}{\mathrm{Kit}_{\mathrm{GF}_{2}}}$ and $\frac{[\mathrm{G}]}{\mathrm{Kit}_{\mathrm{GF}_{3}}}$ in $r_{5}, r_{6}$ and $r_{7}$ respectively are set to zero, whereas the blue line shows inhibition effects. This inhibition affecting FOS dynamics takes place whether or not the reactor is fed and regardless the feeding rate, although it seems to be higher at the beginning of phase 2 . To reduce the inhibition impact due to a high glucose concentration and hence to increase the FOS reaction rate, a glucose concentration constraint is added giving rise to the fed-batch 3 case introduced in the following subsection. In this way, all the constraints defined in Eqs. (7) and (8) are now taken into account.

\subsubsection{Fed-batch 3}

This case departs from the second case by adding the chemical constraint on the glucose concentration. Fig. 7 shows the obtained optimal control input $\hat{Q}_{3}^{*}$ in the upper plot and both the biomass and glucose concentrations in the lower plot. As in the second case, the optimal input can be divided into three phases (vertical black dashed lines in the upper plot of Fig. 7), although in contrast to the first two scenarios the trajectory is considerably different. In the first phase, the optimal control input follows its lower limit $Q_{\min }=0 \mathrm{~L} \mathrm{~h}^{-1}$. This translates into the bioreactor operating in batch mode which allows biomass and glucose concentration to increase as a result of initial sucrose consumption. Since the glucose concentration reaches the boundary of its constraint (red dashed line in the lower plot of Fig. 7), the feed flow rate needs to be adapted to avoid constraint violation. This aspect explains the change in the trajectory of the optimal control input from phase 1 to phase 2 . As in the fed-batch 2 case, Eqs. (30) or (33) can be exploited to theoretically determine the optimal input profile during phase 2 . This phase is characterized by a decrease in the biomass concentration with a constant glucose concentration. Phase 2 lasts until the optimal control input trajectory hits the maximum flow rate constraint $Q_{\max }=0.5 \mathrm{~L} \mathrm{~h}^{-1}$, from which the phase 3 starts. In the latter, the optimal substrate flow rate is kept constant at its upper limit $Q_{\max }$ until the bioreactor is filled at time $\hat{t}_{f 3} \approx 9.7 \mathrm{~h}$. Meanwhile, the biomass concentration keeps decreasing due to the dilution effects. At the end of the considered trajectories both the biomass and the glucose concentrations reach their lower (blue dashed line in the lower plot of Fig. 7) and upper (red dashed line in the lower plot of Fig. 7) constraint limits, respectively.

As detailed in Section 3.4, incompatibility among constraints occurs, which can be observed in Fig. 7. Such phenomenon arises in the control variable due to the occurrence of conflicting constrains. 


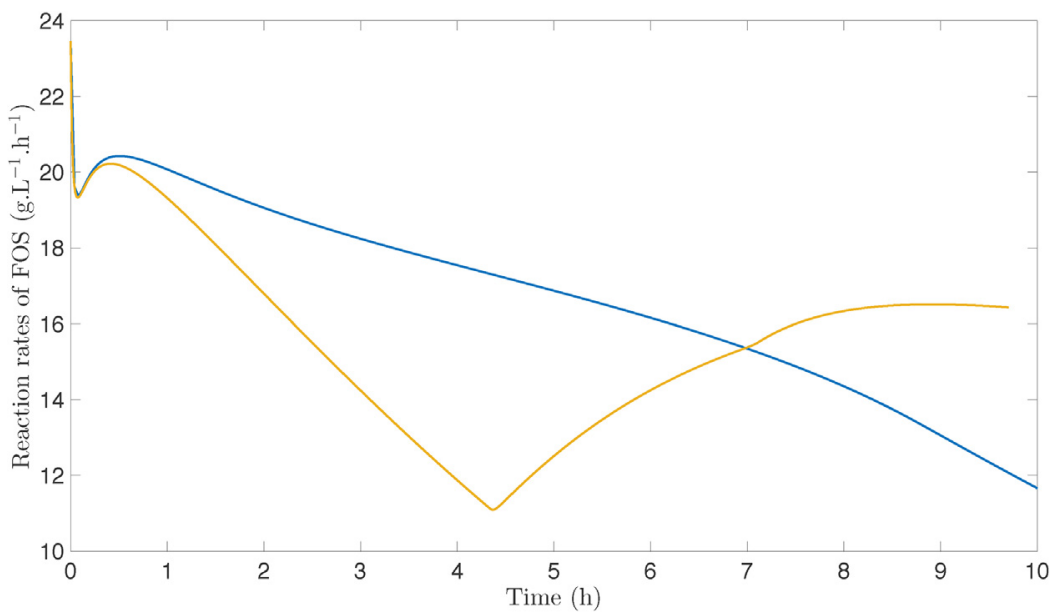

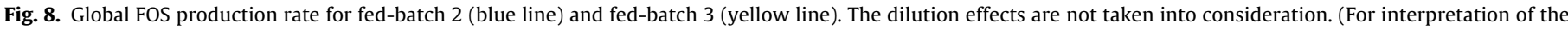
references to color in this figure legend, the reader is referred to the web version of this article.)

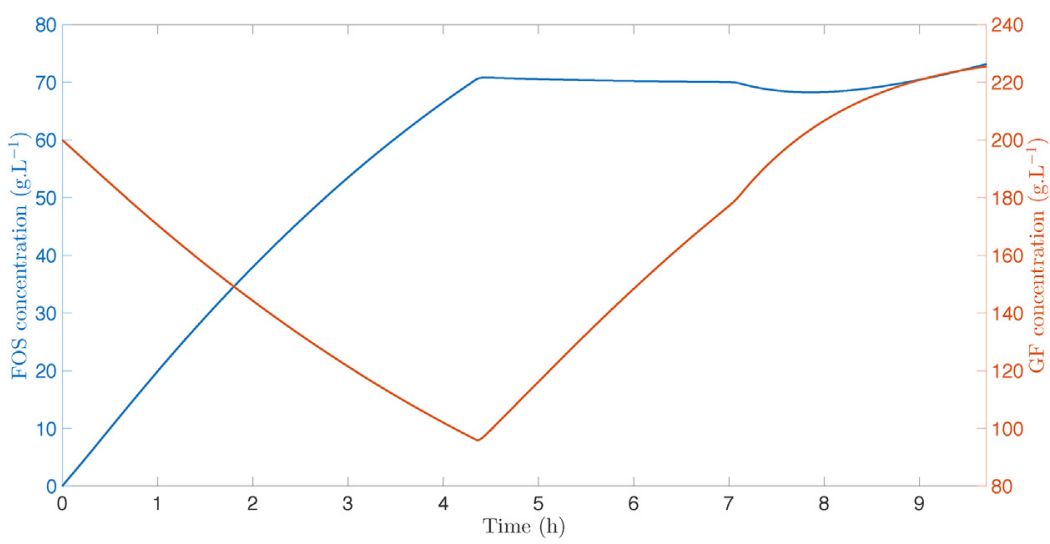

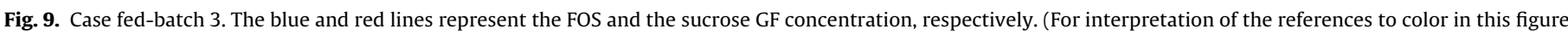
legend, the reader is referred to the web version of this article.)

The required minimal flux value to decrease the glucose concentration is higher than the maximal value to avoid too low enzyme concentration. This corresponds to the situations 2 and 5 indicated at the end of Section 3.4. Consequently, the optimality may not be reached after the determined final time.

Although maximal FOS concentration is hard to achieve when conflicting constraints are considered, a few facts must be highlighted regarding the global FOS production rate shown in Fig. 8. Both fed-batch 2 and 3 are represented in this figure by a blue and yellow lines, respectively. The dilution effect is not taken into consideration in this discussion. Focusing on the fed-batch 3 first, the global FOS production rate gathers the three optimal substrate rate trajectories of the three phases corresponding to the optimal substrate feed rate. Since the input is zero during the first phase $(\approx 4.4 \mathrm{~h})$, a decline in the FOS reaction rate is observed after the first hour. The second and third phases follow where the FOS reaction rate augments along with the FOS concentration, which is linked to the constant glucose concentration imposed by the optimal control input. Considering both fed-batch 2 and 3 now, the FOS reaction rate of fed-batch 3 is bigger than the corresponding one of fed-batch 2 during the third phase. From this analysis follows that controlling the glucose concentration provides a means of increasing the global FOS production rate. Nevertheless, the FOS concentration obtained from fed-batch 3 is lower than the one of fed-batch 2 at the same time instant, i.e. $\mathcal{C}\left(\hat{t}_{f 3}=9.7 \mathrm{~h}\right) \approx 72.4 \mathrm{~g} \mathrm{~L}^{-1}$ and $\mathcal{C}\left(\hat{t}_{f 2}=9.7 \mathrm{~h}\right) \approx 100 \mathrm{~g} \mathrm{~L}^{-1}$ respectively. This is due to the dilution effects that exhibit a stronger influence than the reaction rates.
Moreover, the FOS concentration evolution as well as the sucrose GF for fed-batch 3 are displayed in Fig. 9. It can be seen that the sucrose is not totally consumed in this case explaining the low level of the FOS concentration at the final time.

\subsection{Discussion}

Throughout this section the developed method for dealing with the optimal control problem with state and control constraints has been applied for batch and fed-batch scenarios. Both scenarios, excluding the considered fed-batch 3 case, provide similar results, i.e. an average of $192 \mathrm{~g} \mathrm{~L}^{-1}$ FOS concentration in $30 \mathrm{~h}$. In contrast to the batch case, fed-batch realizations allow to control the enzymatic reactions and the by-products by adapting the substrate feed rate. Different cases have been studied by adding constraints to the problem in order to increase the FOS reaction rate. Case 1 corresponds to constraints associated to basic hardware requirements, namely the feeding profile control. Case 2 builds on case 1 to limit the minimum allowed cell concentration and to keep the enzymatic activity in the medium, whereas case 3 builds on case 2 to limit the maximum glucose concentration in order to avoid the inhibition mechanisms caused by its presence. In this way, hardware limitations are accounted for while providing suitable conditions of cell and glucose concentrations for the chemical reaction to take place. Even if all cases exhibit dilution effects, it is still possible to increase the reaction rates that deliver the FOS components (i.e. $\left.\left[\mathrm{GF}_{2}\right]+\left[\mathrm{GF}_{3}\right]+\left[\mathrm{GF}_{4}\right]\right)$. Furthermore, fed-batch 3 has been devised 
to reduce the glucose inhibition on the FOS production, which increases the global FOS production rate. The latter case, also shows a better yield (see Table 3 ) which represents the effectiveness of the proposed solution to avoid inhibition.

In the three reported case-studies the derived substrate feeding control strategies are performed in open-loop. Although it was outside the scope of this work, a feedback control could also be implemented, where the feeding profiles could be calculated online through the control of some variables, such as, the oxygen uptake rate (OUR), the cellular density or the glucose concentration in the medium. So far, according to the literature, the production through fed-batch fermentation of oligosaccharides or fructosytransferase enzyme, which is responsible for the FOS synthesis, was only performed in open-loop [40-43]. In order to carry out a feedback control for FOS production using Aspergillus sp. cells, two sensors such as $\mathrm{pO}_{2}$ and glucose could be implemented to provide measurements of the OUR and glucose production kinetics throughout the fermentation process. The cellular density of Aspergillus sp. cells would not be a reliable sensor to use as feedback control, as this fungi cells are subject to morphological changes throughout the fermentation process, resulting in pellets form after some time of fermentation, which hampers the biomass determination.

Finally, these results are promising and more experiments should be carried out by studying other operational modes, such as continuous mode or feedback mode. These situations would render the glucose control easier and increase the reaction rates of FOS production and the yield.

\section{Conclusion}

In this paper, an optimal control strategy for the production of fructo-oligosaccharides (FOS) in a fed-batch reactor is developed. To the best of the author's knowledge, this is the first study on FOS optimization considering a mathematical model. The corresponding model is constrained in the control variable to respect the hardware constraints of the bioreactor. Besides, biological and chemical constraints are introduced to keep an acceptable concentration of cells while reducing inhibition. To solve the problem, an optimization procedure based on the Pontryagin's maximum principle is proposed, where the constraints are taken into account by adding penalty functions to the cost function. Since the maximization problem is a singular control problem, a regularization term is used to ensure the concavity conditions of the solution.

This study shows the advantages of using a fed-batch mode to produce FOS by fermentation in a bioreactor. By controlling the input, the proposed strategy allows to bring the experimental conditions to some target conditions at which the reaction process may be improved. These results can be easily extended to continuous mode, where the products are continuously removed and cells wash-out may be avoided by the integration of a filtrate membrane in the reactor outlet. Further work could also examine the effect of modeling uncertainties on the optimal profile, and possibly deduce guidelines on the required model quality.

\section{Acknowledgements}

The authors would like to thank the reviewers for their insightful comments which helped improving significantly the paper.

Luis. D. Couto thanks the Fonds pour la Formation à la Recherche dans l'industrie et dans l'Agriculture (FRIA) of the FNRS for its financial support.

Clarisse Nobre acknowledges the Portuguese Foundation for Science and Technology (FCT) under the scope of the strategic funding of UID/BIO/04469/2019 unit and BioTecNorte operation (NORTE-01-0145-FEDER-000004) funded by the European
Regional Development Fund under the scope of Norte2020 Programa Operacional Regional do Norte also, Project ColOsH 02/SAICT/2017 (POCI-01-0145-FEDER-030071).

\section{References}

[1] V. De Preter, H.M. Hmaer, K. Windey, K. Verbeke, The impact of pre- and/or probiotics on human colonic metabolism: does it affect human health? Mol Nutr. Food Res. 55 (1) (2011) 46-57.

[2] H. Tomomatsu, Health effects of oligosaccharides, Food Technol. 48 (10) (1994) 61-65.

[3] J.W. Yun, Fructooligosaccharides, occurrence, preparation, and application, Enzyme Microb. Technol. 19 (2) (1996) 107-117.

[4] A. Dominguez, C. Nobre, L.R. Rodrigues, A.M. Peres, I. Rocha, N. Lima, J.A. Teixeira, New improved method for fructooligosaccharides production by Aureobasidium pullulans, Carbohydr. Polym. 89 (4) (2012) 1174-1179.

[5] O. Rocha, C. Nobre, A. Dominguez, D. Torres, N. Faria, L. Rodrigues, J.A. Teixeira, E.C. Ferreira, I. Rocha, A dynamical model for the fermentative production of fructooligosaccharides, Comput. Aided Chem. Eng. 27 (2009) 1827-1832.

[6] P.T. Sangeetha, M.N. Ramesh, S.G. Prapulla, Recent trends in the microbial production, analysis and application of fructooligosaccharides, Trends Food Sci. Technol. 16 (10) (2005) 442-457.

[7] J.W. Yun, M.G. Lee, S.K. Song, Batch production of high-content fructo-oligosaccharides from sucrose by the mixed-enzyme system of $\beta$-fructofuranosidase and glucose oxidase, J. Ferment. Bioeng. 77 (2) (1994) 159-163.

[8] I. Rocha, E. Ferreira, Specific growth rate regulation in a simulated fed-batch E. coli fermentation, in: CONTROLO 2006, 7th Portuguese Conference on Automatic Control, Lisboa, 2016.

[9] J.W. Yun, S.K. Song, The production of high-content fructo-oligosaccharides from sucrose by the mixed-enzyme system of fructosyltransferase and glucose-oxidase, Biotechnol. Lett. 15 (6) (1993) 573-576.

[10] C. Nobre, C. Castro, A.-L. Hantson, J. Teixeira, G. De Weireld, L. Rodrigues, Strategies for the production of high-content fructo-oligosaccharides through the removal of small saccharides by co-culture or successive fermentation with yeast, Carbohydr. Polym. 136 (2016) 274-281.

[11] C. Nobre, J.A. Teixeira, L.R. Rodrigues, New trends and technological challenges in the industrial production and purification of fructo-oligosaccharides, Crit. Rev. Food Sci. Nutr. 55 (10) (2015) 1444-1455.

[12] M. Pinelo, G. Jonsson, A.S. Meyer, Membrane technology for purification of enzymatically produced oligosaccharides: molecular and operational features affecting performance, Sep. Purif. Technol. 70 (1) (2009) 1-11.

[13] C. Nobre, J. Teixeira, L. Rodrigues, Fructo-oligosaccharides purification from a fermentative broth using an activated charcoal column, New Biotechnol. 29 (3) (2012) 395-401.

[14] C. Nobre, P. Suvarov, G. De Weireld, Evaluation of commercial resins for fructo-oligosaccharide separation, New Biotechnol. 31 (1) (2014) 55-63.

[15] A. Johnson, The control of fed-batch fermentation processes - a survey, Automatica 23 (6) (1987) 691-705.

[16] K.J. Duan, J.S. Chen, D.C. Sheu, Kinetic studies and mathematical model for enzymatic production of fructooligosaccharides from sucrose, Enzyme Microb. Technol. 16 (4) (1994) 334-339.

[17] R. Fekih-Salem, A. Vande Wouwer, C. De Castro, C. Nobre, A.-L. Hanstson, Parameter identification of the fermentative production of fructo-oligosaccharides by Aureobasidium pullulans, 19th International Conference on System Theory, Control and Computing (2015) 43-48.

[18] K. Jung, J.W. Yun, K.R. Kang, J.Y. Lim, J.H. Lee, Mathematical model for enzymatic production of fructo-oligosaccharides from sucrose, Enzyme Microb. Technol. 11 (8) (1989) 491-494.

[19] D. Bonvin, Optimal operation of batch reactors - a personal view, J. Process Control 8 (5) (1998) 355-368.

[20] J. Lee, S.Y. Lee, S. Park, A.P.J. Middelberg, Control of fed-batch fermentations, Biotechnol. Adv. 17 (1999) 29-48.

[21] L.T. Biegler, Nonlinear Programming: Concepts, Algorithms, and Applications to Chemical Processes, Society for Industrial and Applied Mathematics, 2010.

[22] E. Polak, Computational Methods in Optimization: A Unified Approach, vol. 77, Academic Press, 1971

[23] D.E. Goldberg, Genetic Algorithms in Search, Optimisation and Machine Learning, Addison-Wesley, Reading, New York, 1989.

[24] D. Sarkar, J.M. Modak, Optimization of fed-batch bioreactors using genetic algorithms, Chem. Eng. Sci. 58 (11) (2003) 2283-2296.

[25] E. Aguiar-Oliveira, F. Maugeri, Effects of the addition of substrate and salts in both the fructosyltransferase immobilization and its catalytic properties, J. Food Biochem. 37 (5) (2012) 520-527.

[26] M. Antošová, M. Polakovič, Fructosyltransferases: the enzymes catalyzing production of fructooligosaccharides, Chem. Pap. - Slovak Acad. Sci. 55 (6) (2001) 350-358

[27] J.W. Yun, K.H. Jung, J.W. Oh, J.H. Lee, Semibatch production of fructo-oligosaccharides from sucrose by immobilized cells of aureobasidium pullulans, Appl. Biochem. Biotechnol. 24 (1) (1990) 299-308.

[28] A.E. Bryson, Y.-C. Ho, Applied Optimal Control, Ginn and Co, Waltham, MA 1969. 
[29] I.Y. Smets, J.E. Claes, E.J. November, G.P. Bastin, J.F. Van Impe, Optimal adaptive control of (bio)chemical reactors: past, present and future, J. Process Control 14 (2004) 795-805.

[30] J.F. Van Impe, G. Bastin, Optimal adaptative control of fed-batch fermentation processes, Control Eng. Pract. 3 (7) (1995) 939-954.

[31] L. Shampine, M. Reichelt, J. Kierzenka, Solving boundary value problems for ordinary differential equations in matlab with bvp4c, Tutorial notes, 2000,

Available at http://www.mathworks.com/bvp_tutorial. (Accessed 19 December 2017).

[32] A. Wächter, L.T. Biegler, On the implementation of an interior-point filter line-search algorithm for large-scale nonlinear programming, Math. Program. 106 (1) (2006) 25-57.

[33] T.F. Coleman, Y. Li, An interior trust region approach for nonlinear minimization subject to bounds, SIAM J. Optim. 6 (2) (1996) 418-445.

[34] M. Ahookhosh, K. Amini, M.R. Peyghami, A nonmonotone trust-region line search method for large-scale unconstrained optimization, Appl. Math Model. 36 (1) (2012) 478-487.

[35] D.H. Jacobson, S. Gershwin, M.M. Lele, Computation of optimal singular controls, IEEE Trans. Autom. Control 15 (1) (1970) 67-73.

[36] W. Borzani, Calculation of fermentation parameters from the results of a fed-batch test taking account of the volume of biomass in the fermenting medium, Braz. Arch. Biol. Technol. 51 (3) (2008) 441-446.
[37] S.I. Mussatto, J.A. Teixeira, Increase in the fructooligosaccharides yield andproductivity by solid-state fermentation with Aspergillus japonicus using agro-industrial residues as support and nutrient source, Biochem. Eng. J. 53 (1) (2010) 154-157.

[38] C. Nobre, E. Alves Filho, F. Fernandes, E. Brito, S. Rodrigues, J. Teixeira, L. Rodrigues, Production of fructo-oligosaccharides by Aspergillus ibericus and their chemical characterization, LWT - Food Sci. Technol. 89 (2018) 58-64.

[39] R.C. Fernández, B.G. Maresma, A. Juárez, J. Martínez, Production of fructooligosaccharides by $\beta$-fructofuranosidase from Aspergillus sp 27H, J. Chem. Technol. Biotechnol. 79 (3) (2004) 268-272.

[40] W.C. Chen, C.H. Liu, Production of beta-fructofuranosidase by Aspergillus japonicus, Enzyme Microb. Technol. 18 (2) (1996) 153-160.

[41] J.W. Yun, D.H. Kim, S.K. Song, Enhanced production of fructosyltransferase and glucosyltransferase by substrate-feeding cultures of Aureobasidium pullulans, J. Ferment. Bioeng. 84 (3) (1997) 261-263.

[42] C. Guerrero, C. Vera, A. Illanes, Fed-batch operation for the synthesis of lactulose with $\beta$-galactosidase of Aspergillus oryzae, Bioresour. Technol. 237 (2017) 126-134.

[43] C. Vera, C. Guerrero, A. Illanes, R. Conejeros, Fed-batch synthesis of galacto-oligosaccharides with Aspergillus oryzae $\beta$-galactosidase using optimal control strategy, Biotechnol. Prog. 30 (1) (2014) 59-67. 